Center for

Mathematical Economics

Working Papers

March 2014

Large Deviations and Stochastic Stability in the Small Noise Double Limit, II: The Logit Model

Willian H. Sandholm and Mathias Staudigl

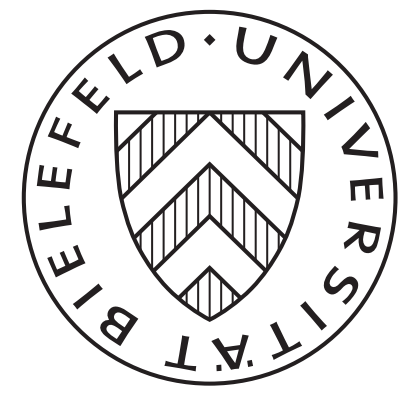




\title{
Large Deviations and Stochastic Stability in the Small Noise Double Limit, II: The Logit Model ${ }^{*}$
}

\author{
William H. Sandholm ${ }^{\dagger}$ and Mathias Staudigl ${ }^{\ddagger}$
}

March 21, 2014

\begin{abstract}
We describe the large deviations properties, stationary distribution asymptotics, and stochastically stable states of stochastic evolutionary processes based on the logit choice rule, focusing on behavior in the small noise double limit. These aspects of the stochastic evolutionary process can be characterized in terms of solutions to certain minimum cost path problems. We solve these problems explicitly using tools from optimal control theory. The analysis focuses on three-strategy coordination games that satisfy the marginal bandwagon property and that have an interior equilibrium, but our approach can be applied to other classes of games and other choice rules.
\end{abstract}

\section{Introduction}

A basic concern of evolutionary game theory is to understand the behavior of populations of strategically interacting agents over long time spans. Doing so means describing how populations escape from and transit between equilibria, and using these descriptions to determine the stochastically stable states, which are played in a large proportion of periods over long enough time spans when suboptimal choices are rare. ${ }^{1}$

Most work on these questions has focused on the best response with mutations (BRM) model of Kandori et al. (1993), in which the probability that an agent plays a suboptimal

${ }^{*}$ We thank Daniel Liberzon for helpful discussions. Financial support from NSF Grants SES-0851580 and SES-1155135 and the Vienna Science and Technology Fund (WWTF) under project fund MA 09-017 is gratefully acknowledged.

${ }^{\dagger}$ Department of Economics, University of Wisconsin, 1180 Observatory Drive, Madison, WI 53706, USA. e-mail: whs@ssc.wisc.edu; website: www.ssc.wisc.edu/ whs.

‡Center for Mathematical Economics, Bielefeld University, Germany. e-mail: mathias.staudigl@ uni-bielefeld.de; website: mwpweb.eu/MathiasStaudigl.

${ }^{1}$ See Foster and Young (1990), Kandori et al. (1993), and Young (1993). 
strategy is independent of its payoff consequences. But in modeling the breakdown of equilibrium, it is sometimes more natural to allow mistake probabilities to depend on their payoff consequences. Under such specifications, the unlikeliness of a path away from equilibrium depends not only on its length, but also on the particular sequence of suboptimal choices the path entails. Because of this complication, little is known about the long term behavior beyond cases with two strategies per player, and the particularly tractable case of potential games under the logit choice rule. ${ }^{2}$

In Sandholm and Staudigl (2014) (henceforth SS), we introduced tools for studying large deviations and stochastic stability in stochastic evolutionary models, evaluating these properties in the small noise double limit. The first limit, which takes the noise level in agent's choice rules to zero, can be analyzed using well-known tools from large deviations theory, ${ }^{3}$ but yields results expressed in terms of solutions to optimal control problems on a discrete state space. These problems are not tractable unless the number of agents is small. In SS, we show that by taking a second limit in the population size, one can approximate the solutions to these discrete control problems by solutions to continuous control problems. Provided that the latter problems can be solved, this approach makes it possible to explicitly describe long term behavior beyond the cases studied to date. However, since the relevant control problems are multidimensional and nonsmooth, it is not obvious that analytical solutions can be obtained.

The aim of the present paper is to show that at least in certain interesting cases, the analysis of large deviations and stochastic stability in the small noise double limit is indeed tractable. Our analysis here focuses on evolution under the logit choice rule (Blume (1993, 1997)), under which the rate of decay of the probability with which a strategy is chosen is determined by the difference between its payoff and that of an optimal strategy. Singling out one interesting class of games, we consider three-strategy coordination games that satisfy the marginal bandwagon property (Kandori and Rob (1998)) and that have an interior equilibrium. This class of games, which we call simple three-strategy coordination games, is large enough to allow a some variety in its analysis, but small enough that the analysis remains manageable. We explain at the close of the paper why similar analyses should be feasible for other classes of games and choice rules.

We analyze the control problems associated with two distinct kinds of large deviations properties. We first consider the exit problem, which is used to assess the expected time until the evolutionary process leaves the basin of attraction of a stable equilibrium, and to determine the likely exit path. Solving this problem for the class of games under

\footnotetext{
${ }^{2}$ For the former, see Blume (2003), Sandholm (2007, 2010b), and Staudigl (2012); for the latter, see Blume (1993, 1997), Alós-Ferrer and Netzer (2010), and Sandholm (2010c, Sec. 11.5).

${ }^{3}$ See Freidlin and Wentzell (1998), Catoni (1999), and Young $(1993,1998)$.
} 
consideration, we show that the likely exit path proceeds along the boundary of the simplex, escaping the basin of attraction through a boundary mixed equilibrium.

To evaluate stationary distribution asymptotics and stochastic stability, one must instead consider the transition problem, which is used to assess the probable time until a transition between a given pair of stable equilibria, and the most likely path that this transition will follow. We solve the transition problem explicitly for simple three-strategy coordination games, and find that the nature of the problem's solution depends in a basic way on whether the game in question is also a potential game. When this is so, the optimal control problem is degenerate, in that there are open sets of states from which there are a continuum of minimal cost paths. Still, the optimal paths between equilibria always proceed directly along the relevant edge of the simplex. The control problem is not degenerate for games without a potential function, which we call skewed games. But unlike in the case of potential games, optimal paths between equilibria of skewed games need not be direct; instead, they may proceed along an alternate edge of the simplex, turn into the interior, and pass through the interior equilibrium.

By combining solutions to the control problems with results from SS, we are able to characterize the long run behavior of the stochastic evolutionary process in the small noise double limit. We use a parameterized class of examples to illustrate the effects of payoff-dependent mistake probabilities on equilibrium selection, and to contrast long-run behavior in the logit and BRM models. In addition, in the class of potential games we consider, we fully describe the asymptotic behavior of the stationary distribution in the small noise double limit, showing that the rate of decay of the stationary distribution mass at each state equals the difference between the value of the potential function at that state and the maximum value of potential. In contrast to those in previous work on logit choice in potential games, ${ }^{4}$ the assumptions we impose on the transition law of the evolutionary process are asymptotic nature, and so do not allow us to express the stationary distribution in closed form. We instead build our analysis on large deviations estimates, and thereby obtain a clearer intuition about the form that the stationary distribution asymptotics take.

The heart of the paper is the analysis of the exit and transition problems. These optimal control problems have nonsmooth running costs, but are rather simple in other respects. If $L(x, u)$ represents the cost of choosing direction of motion $u$ at state $x$, then $L$ is piecewise linear in $u$ regardless of the agents' choice rule. When agents employ the logit choice rule, $L$ is also piecewise linear in $x$.

Taking advantage of these properties, we use sufficient conditions for optimality due to Boltyanskii (1966) and Piccoli and Sussmann (2000) to construct candidate value functions,

\footnotetext{
${ }^{4}$ See Blume $(1993,1997)$ and Sandholm (2010c, Sec. 11.5 and 12.2), as well as Section 7 below.
} 
and to verify that they are indeed the value functions for our problems. These sufficient conditions require the value function to be continuous, to be continuously differentiable except on a finite union of manifolds of positive codimension, and to satisfy the HamiltonJacobi-Bellman equation wherever the value function is smooth. In our case, for each fixed state $x$, the piecewise linearity of $L(x, u)$ in $u$ means that only a small number of controls need to be considered, while the piecewise linearity of $L(x, u)$ in $x$ makes it enough to check the Hamilton-Jacobi-Bellman equation at a small number of well-chosen states.

These properties of the optimal control problem are not dependent on the class of games we consider, but only on the linearity of payoffs in the population state. Moreover, much of the structure of the problem is retained under alternatives to the logit choice rule. Thus as we explain in the final section of the paper, it should be possible to use the approach developed here to study long run behavior in broader classes of games and under other choice rules.

The paper proceeds as follows. Section 2 describes the model of stochastic evolution, defines the class of games we study, and introduces the optimal control problems. Section 3 states the verification theorem and provides some initial steps of the analysis. Section 4 begins the construction of the value functions for the exit and transition problems. Section 5 completes the construction for the exit problem. Section 6 does so for the transition problem, separately considering the cases of potential games and skewed games. Section 7 combines this analysis with results from SS to characterize stationary distribution asymptotics and stochastic stability in the small noise double limit. Section 8 discusses extensions of the analysis to other classes of games and choice rules.

\section{Definitions}

We briefly review the model of stochastic evolution from Sandholm and Staudigl (2014), and introduce new definitions needed here.

\subsection{The stochastic evolutionary model}

A population of size $N$ choose strategies from finite strategy set $S$. The population's aggregate behavior is described by a population state $x$, an element of the grid $X^{N}=X \cap \frac{1}{N} \mathbb{Z}^{n}$ in the simplex $X=\left\{x \in \mathbb{R}_{+}^{n}: \sum_{i=1}^{n} x_{i}=1\right\}$. The standard basis vector $e_{i} \in X \subset \mathbb{R}^{n}$ represents the pure state at which all agents play strategy $i$, and $s(x)=\left\{i \in S: x_{i}>0\right\}$ denotes the support of state $x$. We identify a finite-population game with its payoff function $F^{N}: X^{N} \rightarrow \mathbb{R}^{n}$, where $F_{i}^{N}(x) \in \mathbb{R}$ is the payoff to strategy $i$ when the population state is 
$x \in X^{N}$.

The stochastic evolutionary process $\mathbf{X}^{N, \eta}=\left\{X_{k}^{N, \eta}\right\}_{k=0}^{\infty}$ is a Markov chain with state space $X^{N}$. It is parameterized by the population size $N$ and the noise level $\eta>0$. The index $k$ denotes the number of revision opportunities that have occurred to date, and corresponds to $\frac{k}{N}$ units of clock time. The transition probabilities for the process are given by

$$
P_{x, y}^{N, \eta} \equiv \mathbb{P}\left(X_{k+1}^{N, \eta}=y \mid X_{k}^{N, \eta}=x\right)=x_{i} \sigma_{j}^{\eta}\left(F_{i \rightarrow .}^{N}(x)\right) \text { if } y=x+\frac{1}{N}\left(e_{j}-e_{i}\right), j \neq i,
$$

with the remaining probability assigned to $P_{x, x}^{N, \eta}$. Thus for an agent to switch from strategy $i$ to strategy $j$ during the next period, the agent randomly chosen to revise must be an $i$ player, which occurs with probability $x_{i}$, and this agent must opt to switch to strategy $j$. The probability of the latter is obtained by applying the function $\sigma_{j}^{\eta}$, the $j$ th component of the noisy best response protocol $\sigma^{\eta}: \mathbb{R}^{n} \rightarrow \operatorname{int}(X)$, to $F_{i \rightarrow}^{N}(x) \in \mathbb{R}^{n}$, the clever payoff vector for strategy $i$ players at population state $x$.

In this paper, we take $\sigma^{\eta}$ to be the logit choice protocol, defined by

$$
\sigma_{j}^{\eta}(\pi)=\frac{\exp \left(\eta^{-1} \pi_{j}\right)}{\sum_{k \in S} \exp \left(\eta^{-1} \pi_{k}\right)} .
$$

The clever payoff vector $F_{i \rightarrow \text {. }}^{N}(x) \in \mathbb{R}^{n}$, defined by

$$
F_{i \rightarrow j}^{N}(x)=F_{j}^{N}\left(x+\frac{1}{N}\left(e_{j}-e_{i}\right)\right),
$$

accounts for the slightly different incentives faced by players of different strategies in a finite-population game. We define the pure best response correspondence for strategy $i \in S$ in game $F^{N}$ by

$$
b_{i}^{N}(x)=\underset{j \in S}{\operatorname{argmax}} F_{i \rightarrow j}^{N}(x) .
$$

State $x \in X^{N}$ is a Nash equilibrium of $F^{N}$ if $i \in b_{i}^{N}(x)$ whenever $x_{i}>0$, so that no agent can obtain a higher payoff by switching strategies.

\subsection{Linear population games}

As $N$ grows large, we assume that the finite-population games $F^{N}$ converge uniformly to a limit game $F: X \rightarrow \mathbb{R}^{n}$, which we interpret as a continuous-population game. In this paper this limit game is linear: $F(x)=A x$ for some $A \in \mathbb{R}^{n \times n}$. Games $F^{N}$ and $F$ thus describe 
the matching of agents to play a symmetric normal form game with payoff matrix $A$.

The following notation, while not entirely standard, will be very convenient. We use superscripts to refer to rows of $A$ and subscripts to refer to columns. Thus $A^{i}$ is the $i$ th row of $A, A_{j}$ is the $j$ th column of $A$, and $A_{j}^{i}$ is the $(i, j)$ th entry. These objects can be obtained by pre- and post-multiplying $A$ by standard basis vectors:

$$
A^{i}=e_{i}^{\prime} A, \quad A_{j}=A e_{j}, \quad A_{j}^{i}=e_{i}^{\prime} A e_{j} .
$$

In a similar fashion, we use super- and subscripts of the form $i-j$ to denote certain differences obtained from $A$.

$$
A^{i-j}=A^{i}-A^{j}=\left(e_{i}-e_{j}\right)^{\prime} A, \quad A_{k-\ell}^{i-j}=A_{k}^{i}-A_{\ell}^{i}-A_{k}^{j}+A_{\ell}^{j}=\left(e_{i}-e_{j}\right)^{\prime} A\left(e_{k}-e_{\ell}\right) .
$$

Using this notation, we define the best response region for strategy $i$ by

$$
\mathcal{B}^{i}=\left\{x \in X: A^{i-j} x \geq 0 \text { for all } j \in S\right\}
$$

Similarly, $\mathcal{B}^{i j}=\mathcal{B}^{i} \cap \mathcal{B}^{i}$ describes the boundary between the best response regions for strategies $i$ and $j$.

We call $A$ a coordination game if

$$
A_{i}^{i}>A_{i}^{j} \text { for all } i, j \in S \text { with } j \neq i,
$$

so that each pure state is a Nash equilibrium of $F$. This implies that

$$
A_{i-j}^{i-j}>0 \text { for all } i, j \in S \text {. }
$$

We call $A_{i-j}^{i-j}=A_{j-i}^{j-i}$ the $(i, j)$ th alignment of $A$. This quantity, which corresponds to the denominator of the mixed equilibrium in the binary-choice game with strategies $i$ and $j$, represents the strength of incentives to coordinate (or, if negative, to miscoordinate) in the restricted game with strategy set $\{i, j\}$.

The game $A$ has the marginal bandwagon property (MBP) (Kandori and Rob (1998)) if

$$
A_{i-k}^{i-j}>0 \text { for all } i, j, k \in S \text { with } i \notin\{j, k\} \text {. }
$$

This property requires that when opponents switch to strategy $i$ from some other strategy, the payoffs to playing strategy $i$ improve relative to those of all other strategies.

The next definition for games with three or more strategies plays a basic role in our 
analysis. For an ordered triple of distinct strategies $(i, j, k)$, we define the $(i, j, k)$ th skew of $A$ by

$$
\begin{aligned}
Q^{i j k} & =A_{j-k}^{i}+A_{k-i}^{j}+A_{i-j}^{k} \\
& =A_{i-k}^{i-j}-A_{i-j}^{i-k}=A_{j-i}^{j-k}-A_{j-k}^{j-i}=A_{k-j}^{k-i}-A_{k-i}^{k-j} .
\end{aligned}
$$

Evidently skew is alternating, in the sense that it is preserved by even permutations of the index list and negated by odd ones:

$$
Q^{i j k}=Q^{j k i}=Q^{k i j}=-Q^{k j i}=-Q^{j i k}=-Q^{i k j} .
$$

We call $A$ a potential game if $A=C+1 r^{\prime}$ for some symmetric matrix $C \in \mathbb{R}^{n \times n}$ and some vector $r \in \mathbb{R}^{n}$, where $\mathbf{1} \in \mathbb{R}^{n}$ denotes the vector of ones. Thus $A$ is the sum of a common interest game $C$ and a passive game $\mathbf{1} r^{\prime}$ in which a player's payoff depends only on his opponent's strategy. Clearly, games $A$ and $C$ induce the same best-response correspondence and the same set of Nash equilibria.

Potential games admit a variety of characterizations. For instance, $A$ is a potential game if and only if $\Phi A \Phi$ is a symmetric matrix, where $\Phi=I-\frac{1}{n} \mathbf{1 1}^{\prime} \in \mathbb{R}^{n \times n}$ is the orthogonal projection onto the tangent space $T X=\left\{z \in \mathbb{R}^{n}: \sum_{i} z_{i}=0\right\} .{ }^{5}$ The latter condition says that $A$ is a symmetric bilinear form on $T X \times T X$, meaning that $z^{\prime} A \hat{z}=\hat{z}^{\prime} A z$ for all $z, \hat{z} \in T X$. Alternatively, $A$ is a potential game if and only if it satisfies the triangular integrability condition of Hofbauer (1985), which can be stated in terms of skews: $Q^{i j k}=0$ for all distinct $i, j, k \in S .^{6}$

\subsection{Discrete and complete best response dynamics}

Returning to the evolutionary model, we capture the typical behavior of the process $\mathbf{X}^{N, \eta}$ at low noise levels using the discrete best response dynamic, defined by the difference inclusion

$$
x_{k+1}^{N}-x_{k}^{N} \in\left\{\frac{1}{N}\left(e_{i}-e_{j}\right): j \in s(x) \text { and } i \in b_{j}^{N}(x)\right\} .
$$

We call the set $K^{N} \subseteq X^{N}$ strongly invariant under (7) if no solution to (7) starting in $K^{N}$ ever leaves $K^{N}$. A set that is minimal with respect to this property is called a recurrent class of (7). We denote the collection of such recurrent classes by $\mathcal{K}^{N}$. If $A$ is a coordination game

${ }^{5}$ The "only if" direction of this claim is obvious. Letting $\Xi=\frac{1}{n} \mathbf{1 1}^{\prime}=I-\Phi$, the "if" direction follows from the decomposition $A=\left(\Phi A \Phi+\left(\Phi A \Xi+\Xi A^{\prime} \Phi\right)\right)+\Xi\left(A-A^{\prime} \Phi\right)$. Compare Sandholm (2010a).

${ }^{6}$ See Sandholm (2009, Proposition 4.5). 
with the marginal bandwagon property, then $\mathcal{K}^{N}=\mathcal{K}=\left\{\left\{e_{1}\right\}, \ldots,\left\{e_{n}\right\}\right\}$, so that recurrent classes correspond to strict equilibria. ${ }^{7}$

The limiting version of (7) is the complete best response dynamic. This dynamic is defined as the differential inclusion

$$
\dot{x} \in \operatorname{conv}\left(\left\{e_{i}-e_{j}: j \in s(x), i \in b(x)\right\}\right),
$$

where $b(x)=\operatorname{argmax}_{i \in S} F_{i}(x)$ is the pure best response correspondence of the limit game $F$. An absolutely continuous path $\phi:[0, T] \rightarrow X$ path is a solution of (8) if the inclusion in (8) holds at almost all $t \in[0, T]$.

For a coordination game $A$, the weak basin of attraction $\mathcal{W}\left(e_{i}\right)$ of pure equilibrium $e_{i}$ under the complete best response dynamic consists of those states from which there is a solution of (8) that terminates at $e_{i}$. $\mathcal{W}\left(e_{i}\right)$ contains the best response region $\mathcal{B}^{i}$ : since $\mathcal{B}^{i}$ is convex and contains $e_{i}$, there is a solution of (8) from each state in $\mathcal{B}^{i}$ that proceeds in a straight line to $e_{i}$. The strong basin of attraction $S\left(e_{i}\right)$ of $e_{i}$ is the set of states from which no solution of (8) terminates at a pure equilibrium besides $e_{i}$. It follows immediately that

$$
\mathcal{S}\left(e_{i}\right)=X \backslash \bigcup_{j \neq i} \mathcal{W}\left(e_{j}\right) .
$$

\subsection{Path costs}

Our aim in this paper is to understand how the process $\mathbf{X}^{\mathrm{N}, \eta}$ escapes from and transits between recurrent states, and to use this information to describe stationary distributions and to evaluate stochastic stability. To obtain analytical results, we consider the process as the parameters $N$ and $\eta$ are taken to their limiting values. For the small noise limit, wellknown results from large deviations theory (Freidlin and Wentzell (1998), Catoni (1999), Young (1998)) allow one to describe long-term behavior in terms of certain minimum cost paths through the discrete space $X^{N}$. In SS, we show that as the population size $N$ grows large, these discrete control problems converge to continuous ones, defined in terms of continuous paths through the simplex $X$.

To define the latter problems, we introduce the unlikelihood function $\Upsilon: \mathbb{R}^{n} \rightarrow \mathbb{R}_{+}^{n}$ associated with the protocols $\left\{\sigma^{\eta}\right\}_{\eta>0}$ :

$$
\Upsilon_{j}(\pi)=-\lim _{\eta \rightarrow 0} \eta \log \sigma_{j}^{\eta}(\pi) .
$$

The cost of absolutely continuous path $\phi:[0, T] \rightarrow X$ is its aggregate unlikelihood:

\footnotetext{
${ }^{7}$ See Kandori and Rob (1998) or SS Example 3.1.
} 


$$
c(\phi)=\int_{0}^{T}\left[\dot{\phi}_{t}\right]_{+}^{\prime} \Upsilon\left(F\left(\phi_{t}\right)\right) \mathrm{d} t .
$$

Under any noisy best response protocol, a path has zero cost if and only if it is a solution of the complete best response dynamic (8).

The unlikelihood function for the logit choice protocol (1) is

$$
\Upsilon_{i}(\pi)=\max _{j \in S} \pi_{j}-\pi_{i}
$$

Thus the cost function under this protocol for the linear game $F(x)=A x$ is

$$
c(\phi)=\int_{0}^{T}\left[\dot{\phi}_{t}\right]_{+}^{\prime}\left(\mathbf{1} A^{\check{b}\left(\phi_{t}\right)}-A\right) \phi_{t} \mathrm{~d} t,
$$

where $\breve{b}(\cdot)$ is any selection from the game's pure best response correspondence $b(\cdot)$.

\subsection{The minimum cost path problems}

Two kinds of minimum cost path problems are used to describe the long-term behavior of the process $\mathbf{X}^{N, \eta}$. To introduce them, we let $\Phi(K, L)$ denote the set of absolutely continuous paths through $X$ from the closed set $K \subset X$ to the closed set $L \subset X$.

The cost of exit from the strong basin of attraction (9) of strict equilibrium $e_{i}$ is defined by the following problem:

$$
C\left(\left\{e_{i}\right\}, \cup_{j \neq i} \mathcal{W}\left(e_{j}\right)\right)=\min \left\{c(\phi): \phi \in \Phi\left(\left\{e_{i}\right\}, \cup_{j \neq i} \mathcal{W}\left(e_{j}\right)\right)\right\}
$$

The cost of exit describes the asymptotic behavior of the expected time before the process escapes the strong basin of attraction of $e_{i}$. Suppose we run the process $\mathbf{X}^{N, \eta}$ from state $e_{i}$, and let $\tau^{N, \eta}=\min \left\{k: X_{k}^{N, \eta}=e_{j}\right.$ for some $\left.j \neq i\right\}$ be the time at which the process first reaches some other strict equilibrium. Then SS Corollary 6.1 implies that

$$
\lim _{N \rightarrow \infty} \lim _{\eta \rightarrow 0} \frac{\eta}{N} \log \mathbb{E}\left[\tau^{N, \eta} \mid X_{0}^{N, \eta}=e_{i}\right]=C\left(\left\{e_{i}\right\}, \cup_{j \neq i} \mathcal{W}\left(e_{j}\right)\right) \text {. }
$$

In addition, the cost minimizing path in (14) indicates the point on the strong basin's boundary by which the escape route is likely to pass.

To account for the global behavior of the process $\mathbf{X}^{N, \eta}$-specifically, to determine the expected time until a specific equilibrium $e_{k}$ is reached, and to evaluate stationary distributions and stochastic stability - we must consider the cost of a transition from one strict 
equilibrium $e_{i}$ to the weak basin of attraction of another:

$$
C\left(\left\{e_{i}\right\}, \mathcal{W}\left(e_{k}\right)\right)=\inf \left\{c(\phi): \phi \in \Phi\left(\left\{e_{i}\right\}, \mathcal{W}\left(e_{k}\right)\right)\right\}
$$

Characterizations of the global behavior of $\mathbf{X}^{\mathrm{N}, \eta}$ use the transition costs (15) in combination with graph theoretic arguments; see Section 7 below.

We noted above that when $A$ is a coordination game, $\mathcal{B}^{k} \subseteq \mathcal{W}\left(e_{k}\right)$. It follows that the minimum cost path problems (14) and (15) do not change if we replace $\mathcal{W}\left(e_{k}\right)$ with $\mathcal{B}^{k}$, since one can travel from $\mathcal{W}\left(e_{k}\right)$ to $\mathcal{B}^{k}$ at zero cost. It will be convenient to do so from this point forward. Actually, it will be clear from the analysis below that in the class of games we consider, $\mathcal{W}\left(e_{k}\right)$ and $\mathcal{B}^{k}$ are identical.

\section{Preliminary Analysis}

To understand the long-term behavior of the processes $\mathbf{X}^{N, \eta}$ in the small noise double limit, we must solve the exit cost problems (14) and the transition cost problems (15). These problems have nonsmooth running costs, and are multidimensional in games with more than two strategies. The main task of the present paper is to show that the problems can nevertheless be solved explicitly. We focus on a class of three-strategy coordination games and on the logit choice protocol, but we explain in Section 8 why solutions can also be obtained in other settings.

\subsection{A verification theorem}

We now introduce the result from optimal control theory that we use to solve the cost minimization problems above.

Let $\mathcal{A}$ be an $m$-dimensional affine subspace of $\mathbb{R}^{n}$ with tangent space $T \mathcal{A}$, and let the set $\Omega \subset \mathcal{A}$ be closed relative to $\mathcal{A}$ and have piecewise smooth boundary. Let the function $L: \mathcal{A} \times T \mathcal{A} \rightarrow \mathbb{R}_{+}$be Lipschitz continuous, and let $Z \subset T \mathcal{A}$ be compact and convex. The control problem and its value function $V^{*}: \mathcal{A} \rightarrow \mathbb{R}_{+}$are defined as follows:

$$
\begin{array}{ll}
V^{*}(x)=\min & \int_{0}^{T} L\left(\phi_{t}, v_{t}\right) \mathrm{d} t \\
\text { over } & T \in[0, \infty), v:[0, T] \rightarrow Z \text { measurable } \\
\text { subject to } & \phi:[0, T] \rightarrow \mathcal{A} \text { absolutely continuous, } \\
& \phi_{0}=x, \phi_{T} \in \Omega,
\end{array}
$$




$$
\dot{\phi}_{t}=v_{t} \text { for almost every } t \in[0, T] .
$$

Theorem 3.1 provides sufficient conditions for a function $V: \mathcal{A} \rightarrow \mathbb{R}_{+}$to be the value function of (16). The key requirement is that the Hamilton-Jacobi-Bellman (HJB) equation

$$
\min _{u \in \mathcal{Z}}(L(x, u)+D V(x) u)=0
$$

hold at almost every $x \in \mathcal{A}$.

Theorem 3.1 (Verification theorem (Boltyanskii (1966), Piccoli and Sussmann (2000))).

Let $V: \mathcal{A} \rightarrow \mathbb{R}_{+}$be a continuous function that is continuously differentiable except on the union $\mathcal{U} \subset \mathcal{A}$ of a finite number of manifolds, each of dimension less than $m$. Suppose that

(i) For every $x \in \mathcal{A}$, there is a time $T \in[0, \infty)$ and a measurable function $v:[0, T] \rightarrow Z$ such that the corresponding controlled trajectory $\phi:[0, T] \rightarrow \mathcal{A}$ with $\phi_{0}=x$ satisfies $\phi_{T} \in \Omega$ and $\int_{0}^{T} L\left(\phi_{t}, v_{t}\right) \mathrm{d} t=V(x)$

(ii) The HJB equation (17) holds at all $x \in \mathcal{A} \backslash \mathcal{U}$. Then $V=V^{*}$.

Condition ( $i$ ) of the theorem says that the values specified by the function $V$ can all be achieved, and so implies that $V^{*} \leq V$. Establishing the opposite inequality is straightforward if $V$ is $C^{1}$. Suppose that this is the case, and that $\hat{T} \in[0, \infty)$ and $\hat{v}:[0, \hat{T}] \rightarrow$ $Z$ are feasible in problem (16), so that the controlled trajectory $\hat{\phi}:[0, \hat{T}] \rightarrow \mathcal{A}$ with $\hat{\phi}_{0}=x$ satisfies $\hat{\phi}_{\hat{T}} \in \Omega$. Then the HJB equation (17) implies that

$$
L\left(\hat{\phi}_{t}, \hat{v}_{t}\right) \geq-D V\left(\hat{\phi}_{t}\right) \hat{v}_{t}=-\frac{\mathrm{d}}{\mathrm{d} t} V\left(\hat{\phi}_{t}\right) \text { for almost all } t \in(0, \hat{T}) .
$$

Integrating yields

$$
\int_{0}^{\hat{T}} L\left(\phi_{t}, v_{t}\right) \geq-\left(V\left(\hat{\phi}_{\hat{T}}\right)-V\left(\hat{\phi}_{0}\right)\right)=V(x),
$$

and so $V^{*} \geq V$.

To prove Theorem 3.1 as stated, one establishes that the cost of any feasible controlled trajectory can be approximated arbitrarily well by the cost of a feasible controlled trajectory that only intersects the manifolds in $\mathcal{U}$ at a finite set of times. The first result along these lines is due to Boltyanskii (1966), with various improvements culminating in the work of Piccoli and Sussmann (2000). Theorem 3.1 above follows from the statement and proof of Theorem 6.3.1 in the textbook treatment of Schättler and Ledzewicz (2012). ${ }^{8}$

\footnotetext{
${ }^{8}$ In the statement of Theorem 6.3.1 of Schättler and Ledzewicz (2012), $\mathcal{A}$ is all of $\mathbb{R}^{n}$, the function $L$ is $C^{1}$,
} 


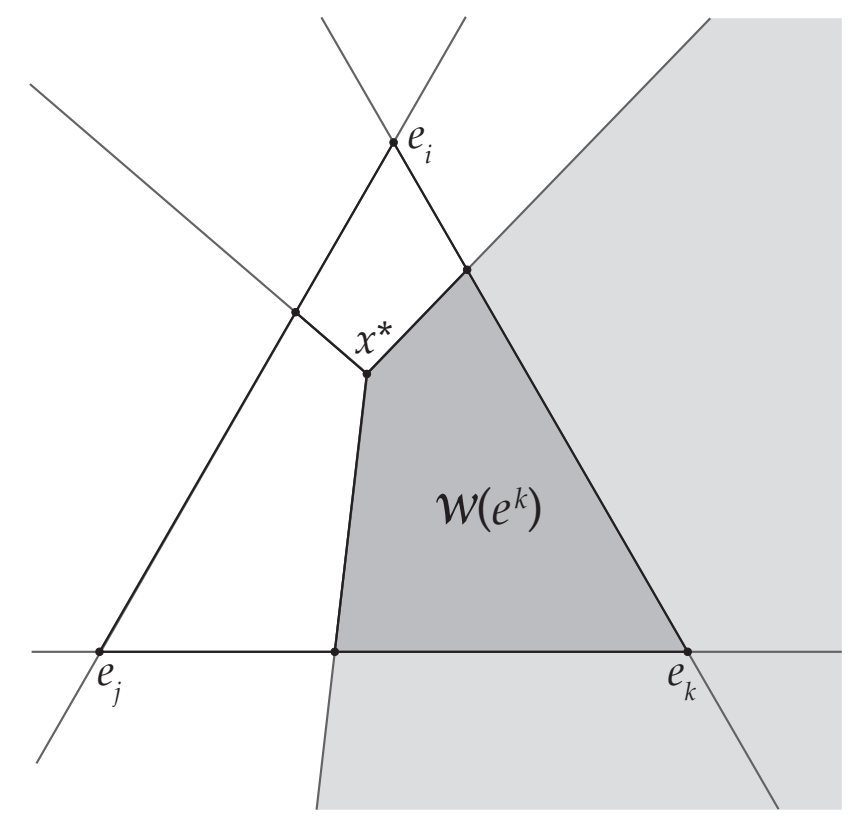

Figure 1: The original and extended versions of the transition problem (15).

While our control problems are set in the simplex $X$, Theorem 3.1 addresses problems whose state space is an affine subset of $\mathbb{R}^{n}$. To use the theorem, we redefine our problems by extending their state space to the affine hull aff $(X)=\left\{x \in \mathbb{R}^{n}: \sum_{i} x_{i}=1\right\}$ of $X$. Since our target sets are defined by linear inequalities, we can define the target sets of our extended problem by imposing the same linear inequalities in aff $(X)$ rather than in $X$ (Figure 1$)$. If in this extended problem, the optimal paths from initial conditions in $X$ to the extended target set are themselves contained in $X$, then these paths are optimal in the original problem; consequently, the restriction of the resulting value function to $X$ is the value function of the original problem. This is precisely what happens in the minimum cost path problems for the games we focus on here. We discuss the general case in Section 8.

Also, notice that under path cost function (11), reparameterizing a path-changing the speed at which the states in the path are traversed-does not affect its cost. Thus in looking for minimum cost paths between sets in aff $(X)$, it is without loss of generality to consider paths satisfying $\dot{\phi}_{t} \in Z$, where $Z$ is the compact set $\operatorname{conv}\left(\left\{e_{i}-e_{j}: i, j \in S\right\}\right)$.

\subsection{A lemma for checking the HJB equation}

We now introduce a basic tool for verifying the HJB equation in our setting. When $x$ is in $\mathcal{B}^{i} \subset$ aff $(X)$, the HJB equation (17) becomes

and the target set $\Omega$ is required to have smooth boundary. However, inspection of their proof reveals that it goes through unchanged under the weaker requirements imposed in Theorem 3.1 above. 


$$
\min _{u \in Z}\left([u]_{+}^{\prime}\left(\mathbf{1} A^{i}-A\right) x+D V(x) u\right)=0 .
$$

Since the function being minimized in (18) is linear in $u$ on each orthant of $\mathbb{R}^{n}$, there must be a minimizer either at an extreme point of $Z$ or at the origin, where the function evaluates to 0 . Therefore, substituting $e_{b}-e_{a}$ for $u$, we see that (18) is equivalent to

$$
\min _{e_{a}, e_{b} \neq e_{a}}\left(\left(e_{i}-e_{a}\right)^{\prime} A x+D V(x)\left(e_{a}-e_{b}\right)\right) \geq 0 .
$$

Lemma 3.2 provides a sufficient condition for the HJB equation (19) to be satisfied at a state in the (relative) interior of $\mathcal{B}^{i}$ when $A$ is a three-strategy game.

Lemma 3.2. Let $A$ be a three-strategy game with $S=\{i, j, k\}$. Suppose that the candidate value function $V$ is constructed from a feedback control that takes value $e_{k}-e_{i}$ at all states in a neighborhood of $x \in \operatorname{int}\left(\mathcal{B}^{i}\right)$. If

$$
\begin{aligned}
& D V(x)\left(e_{i}-e_{h}\right) \geq 0 \text { for } h \in\{j, k\}, \text { and } \\
& \left(D V(x)-(A x)^{\prime}\right)\left(e_{j}-e_{k}\right) \geq 0,
\end{aligned}
$$

then $V$ satisfies the HJB equation (19) at $x$.

The proof of Lemma 3.2 is presented in Appendix A.1. We argue there that the assumption that the control is $e_{k}-e_{i}$ in a neighborhood of $x$ implies that the function to be minimized in the HJB equation (19) equals 0 when $e_{a}=e_{k}$ and $e_{b}=e_{i}$. This equality can be restated as

$$
\left(D V(x)-(A x)^{\prime}\right)\left(e_{k}-e_{i}\right)=0 .
$$

The proof then uses conditions (20)-(22), and the fact that $x \in \mathcal{B}^{i}$ to show that the function to be minimized in (19) is nonnegative for the remaining five choices of $e_{a}-e_{b}$.

\subsection{Costs of direct paths}

As a final preliminary, we present two simple formulas for path costs in linear games under the logit rule. For $x, y \in \operatorname{aff}(X)$, we let $\gamma(x, y)$ denote the cost of the direct (straightline) path from $x$ to $y$ :

$$
\gamma(x, y)=c(\phi), \text { where } \phi:[0,1] \rightarrow X \text { is defined by } \phi_{t}=(1-t) x+t y \text {. }
$$

The first formula concerns a class of direct paths whose costs are easily expressed in terms of the paths' endpoints: those in which the motion of the state involves agents switching 
away from the current best response.

Lemma 3.3. Suppose that $x, y \in \mathcal{B}^{i}$, and that $y-x=d\left(\alpha-e_{i}\right)$ for some $\alpha \in X$ with $\alpha_{i}=0$ and some $d \geq 0$. Then

$$
\gamma(x, y)=(x-y)^{\prime} A\left(\frac{x+y}{2}\right)
$$

Proof. Since $\dot{\phi}_{t}=y-x=d\left(\alpha-e_{i}\right) \in T X$ and $\left[\dot{\phi}_{t}\right]_{-}^{\prime}\left(\mathbf{1} A^{i}-A\right)=d e_{i}^{\prime}\left(\mathbf{1} A^{i}-A\right)=d\left(A^{i}-A^{i}\right)=\mathbf{0}^{\prime}$,

$$
\begin{aligned}
\gamma(x, y) & =\int_{0}^{1}\left[\dot{\phi}_{t}\right]_{+}^{\prime}\left(\mathbf{1} A^{i}-A\right) \phi_{t} \mathrm{~d} t=\int_{0}^{1} \dot{\phi}_{t}^{\prime}\left(\mathbf{1} A^{i}-A\right) \phi_{t} \mathrm{~d} t=-\int_{0}^{1} \dot{\phi}_{t}^{\prime} A \phi_{t} \mathrm{~d} t \\
& =-(y-x)^{\prime} A \int_{0}^{1} \phi_{t} \mathrm{~d} t=(x-y)^{\prime} A\left(\frac{x+y}{2}\right) .
\end{aligned}
$$

In some important cases this formula can be simplified further. The second formula describes the costs that are realized when the state moves from $x \in \mathcal{B}^{i}$ in direction $e_{k}-e_{i}$ until reaching a state $y$ in the set $\mathcal{B}^{i j}=\mathcal{B}^{i} \cap \mathcal{B}^{j}$, where strategies $i$ and $j$ are both optimal.

Lemma 3.4. Let $x \in \mathcal{B}^{i}$, and suppose that

$$
y=x+d\left(e_{k}-e_{i}\right) \in \mathcal{B}^{i j} \text { for some } d>0
$$

and that $A_{i-k}^{i-j} \neq 0$. Then

$$
\begin{aligned}
& d=\frac{A^{i-j} x}{A_{i-k}^{i-j}} \text { and } \\
& \gamma(x, y)=d A^{i-k} y+\frac{1}{2} d^{2} A_{i-k}^{i-k} .
\end{aligned}
$$

In particular, if $j=k$, then $A^{i-k} y=0$, so (25) becomes

$$
\gamma(x, y)=\frac{1}{2} d^{2} A_{i-k}^{i-k}=\frac{1}{2} \frac{\left(A^{i-k} x\right)^{2}}{A_{i-k}^{i-k}} .
$$

Proof. Since $y \in \mathcal{B}^{i j}, A^{i-j} y=0$, which with (23) implies (24). Also, combining (23) and Lemma 3.3 yields (25), since

$$
\gamma(x, y)=(x-y)^{\prime} A\left(\frac{x+y}{2}\right)=d\left(e_{i}-e_{k}\right)^{\prime} A\left(y+\frac{1}{2} d\left(e_{i}-e_{k}\right)\right)=d A^{i-k} y+\frac{1}{2} d^{2} A_{i-k}^{i-k}
$$




\section{Construction of Value Functions: The Initial Step}

\subsection{Simple three-strategy coordination games}

We now focus on three-strategy coordination games (2) that satisfy the marginal bandwagon property (4) and that admit a completely mixed equilibrium, a class of games we henceforth call simple three-strategy coordination games. The completely mixed equilibrium $x^{*} \in \operatorname{int}(X)$ is the unique state in $\operatorname{aff}(X)$ at which the payoffs to all strategies are equal: $A x^{*}=c \mathbf{1}$ for some $c \in \mathbb{R}$. For distinct strategies $i, j \in S$, such games admit a unique mixed equilibrium $x^{i j}$ with support $\{i, j\}$. This $x^{i j}$ is the unique state in $\mathcal{B}^{i j}$ with $x_{k}=0$.

We now define two vectors that play basic roles in the analysis to come. For distinct strategies $i, j \in S$, we define the vector $\zeta^{i j}=\zeta^{j i} \in T X$ by

$$
\zeta^{i j}=\frac{1}{x_{k}^{*}}\left(x^{i j}-x^{*}\right)
$$

When drawn with its tail at $x^{*}, \zeta^{i j}$ points outward along the boundary $\mathcal{B}^{i j}$ between best response regions $\mathcal{B}^{i}$ and $\mathcal{B}^{j}$ (Figure 2). Since the vector $\left(A^{j-i}\right)^{\prime}$ is normal to $\mathcal{B}^{i j}$, $\zeta^{i j}$ is a multiple of the cross product

$$
\left(A^{j-i}\right)^{\prime} \times \mathbf{1}=A_{j-k}^{j-i} e_{i}+A_{i-k}^{i-j} e_{j}-A_{j-i}^{j-i} e_{k} .
$$

Since (27) implies that $\zeta_{k}^{i j}=-1$, it follows that

$$
\zeta^{i j}=\frac{A_{j-k}^{j-i}}{A_{j-i}^{j-i}} e_{i}+\frac{A_{i-k}^{i-j}}{A_{j-i}^{j-i}} e_{j}-e_{k} \equiv \beta^{i j}-e_{k} .
$$

The equivalence in (28) defines the vector $\beta^{i j}$. Since $A$ is a coordination game with the marginal bandwagon property, $\beta^{i j}$ is an element of $\operatorname{conv}\left(\left\{e_{i}, e_{j}\right\}\right)$, and so $\zeta^{i j}$ is a convex combination of $e_{i}-e_{k}$ and $e_{j}-e_{k}$, as shown in Figure 2.

Next, we define the vector $v^{i j}=v^{j i} \in \mathbb{R}^{3}$ by

$$
\left(v^{i j}\right)^{\prime}=\left(\zeta^{i j}\right)^{\prime} A=\frac{1}{A_{j-i}^{j-i}}\left(A_{j-k}^{j-i} A^{i}+A_{i-k}^{i-j} A^{j}-A_{j-i}^{j-i} A^{k}\right) .
$$

By definition (27) of $\zeta^{i j},\left(v^{i j}\right)^{\prime} x$ is positive if and only if mixed strategy $x^{i j}$ earns a higher payoff than mixed strategy $x^{*}$ at state $x$. Both the geometry and the importance of the vector $v^{i j}$ will be made clear below. 


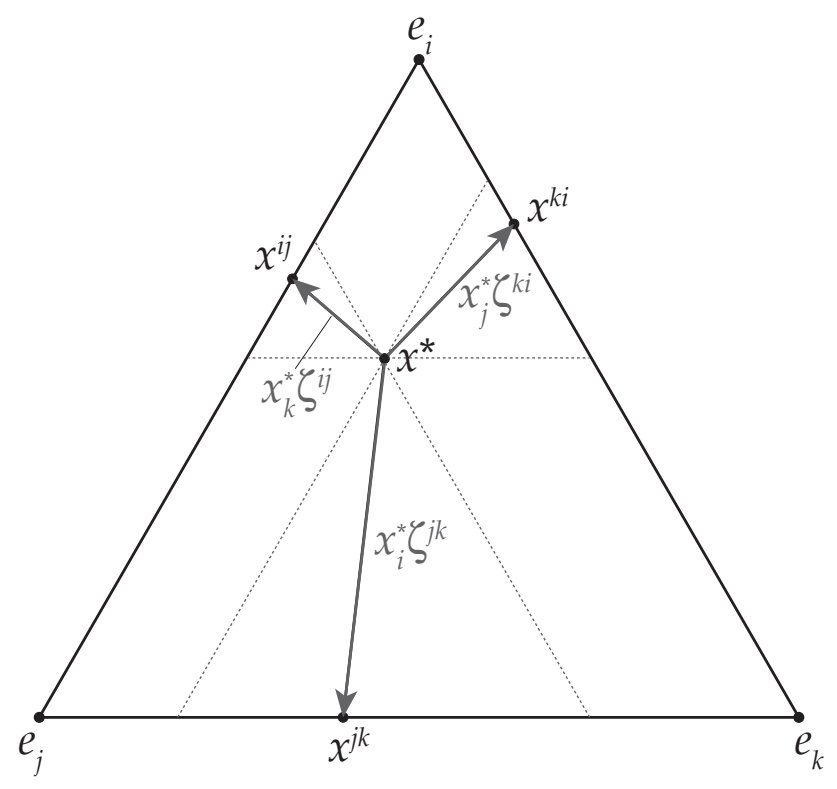

Figure 2: Multiples of the vectors $\zeta^{i j}, \zeta^{j k}$, and $\zeta^{k i}$.

\subsection{Construction of the value function near the target set}

To solve the exit cost problem (14) and the transition cost problem (15) via dynamic programming, we first determine the form of the value function at states near the target set. We therefore consider the cost of reaching the set $\mathcal{B}^{k}$ from nearby states in $\mathcal{B}^{i}$. It is natural to guess that there is a region $R^{i k} \subseteq \mathcal{B}^{i}$ whose boundary contains $\mathcal{B}^{i k}$ in which motion in direction $e_{k}-e_{i}$ leads to $\mathcal{B}^{i k}$, and in fact defines the optimal feedback control. By Lemma 3.4, this choice of control generates the candidate value function

$$
V(x)=\frac{1}{2} \frac{\left(A^{i-k} x\right)^{2}}{A_{i-k}^{i-k}}
$$

in region $R^{i k}$.

We use Lemma 3.2 to determine when this function satisfies the HJB equation (19) in $R^{i k}$. To start, we compute the derivative ${ }^{9} D V: \operatorname{aff}(X) \rightarrow L(T X, \mathbb{R})$ of $V$ at points in the interior of $R^{i k}$ :

$$
D V(x) z=\frac{A^{i-k} x}{A_{i-k}^{i-k}} A^{i-k} z \text { for } x \in \operatorname{int}\left(R^{i k}\right) .
$$

${ }^{9}$ Since $V$ is defined on aff $(X)$, its derivative at $x, D V(x)$, is a linear map from $T X$ to $\mathbb{R}$. There are many vectors $v \in \mathbb{R}^{n}$ that represent this map, in the sense that $D V(x) z=v^{\prime} z$ for all $z \in T X$. The gradient of $V$ at $x, \nabla V(x)$, is the defined to be the unique representative of $D V(x)$ in $T X$; it can be obtained by applying the orthogonal projection matrix $\Phi=I-\frac{1}{n} \mathbf{1 1}^{\prime}$ to an arbitrary representative of $D V(x)$ in $\mathbb{R}^{n}$. See Sandholm (2010c, Section 3.C) for further discussion. 
Since strategies $i$ and $k$ are both best responses at states in $\mathcal{B}^{i k}$, vectors tangent to $\mathcal{B}^{i k}$ are orthogonal to $\left(A^{i-k}\right)^{\prime}$. Equation (31) implies that such vectors $\hat{z}$ satisfy $D V(x) \hat{z}=0$, and so are tangent to the level sets of the value function. Intuitively, moving the state in a direction tangent to $\mathcal{B}^{i k}$ changes neither the distance needed to travel to $\mathcal{B}^{i k}$ nor the payoff differences that must be overcome en route.

We now apply Lemma 3.2. To check condition (20), we first observe that

$$
D V(x)\left(e_{i}-e_{h}\right)=\frac{A^{i-k} x}{A_{i-k}^{i-k}} A_{i-h}^{i-k} .
$$

Now $A^{i-k} x \geq 0$ (since $x \in \mathcal{B}^{i}$ ), $A_{i-k}^{i-k}>0$ (since $A$ is a coordination game; see (3)), and $A_{i-j}^{i-k} \geq 0$ (by the marginal bandwagon property (4)). Thus $D V(x)\left(e_{i}-e_{h}\right) \geq 0$ for $h \in\{j, k\}$, establishing condition (20). To check condition (21), we compute as follows:

$$
\begin{aligned}
\left(D V(x)-(A x)^{\prime}\right)\left(e_{j}-e_{k}\right) & =\frac{A^{i-k} x}{A_{i-k}^{i-k}} A_{j-k}^{i-k}-A^{j-k} x \\
& =\frac{1}{A_{i-k}^{i-k}}\left(A_{k-j}^{k-i} A^{i-k}-A_{k-i}^{k-i} A^{j-k}\right) x \\
& =\frac{1}{A_{i-k}^{i-k}}\left(A_{k-j}^{k-i} A^{i}-A_{k-i}^{k-i} A^{j}+A_{i-j}^{i-k} A^{k}\right) x \\
& =\left(v^{k i}\right)^{\prime} x .
\end{aligned}
$$

Lemma 3.2 thus yields the following result:

Lemma 4.1. Suppose that the function $V$ is defined by equation (30) on a region $R^{i k} \subseteq \mathcal{B}^{i}$ as specified above. Then the HJB equation (19) for $V$ is satisfied at $x \in \operatorname{int}\left(R^{i k}\right)$ if

$$
\left(v^{k i}\right)^{\prime} x \geq 0
$$

By our earlier interpretation of $v^{k i}$, inequality (34) requires that at state $x$, mixed strategy $x^{k i}$ is a weakly better response than mixed strategy $x^{*}$.

\subsection{The geometry of the initial sufficient condition}

We now describe the necessary condition (34) from Lemma 4.1 in geometric terms. In what follows, it is convenient to endow the strategy set $S=\{1,2,3\}$ with the cyclic order $1<2<3<1$. When we refer to the strategies generically, as $i, j$, and $k$, we require that this labeling satisfy $i<j<k<i$. We give the order a geometric meaning by labeling 
the vertices of the simplex $X$ counterclockwise, as in Figure 2. If $\mathbb{R}^{3}$ is presented in righthanded coordinates, so that the cross product obeys the right-hand rule, then our labeling of $X$ corresponds to the view from the "outside", with the origin lying behind the figure, and the vector 1 pointing towards us.

Also, recalling the definition (5) and alternating property (6) of the skew, we abuse notation by writing $Q=Q^{i j k}=-Q^{k j i}$. It follows from the discussion in Section 2.2 that the three-strategy games with zero skew are the potential games. Games with $Q>0$ and $Q<0$ are said to have clockwise skew and counterclockwise skew. ${ }^{10}$ Since the sign of the skew can be reversed by renaming the strategies, there is no loss of generality in focusing on games with zero or clockwise skew.

The following properties of the normal vector $v^{k i}$ allow us to locate the states satisfying inequality (34), and hint at the effects of skew on solutions of our optimal control problems. It follows from expression (32) for $v^{k i}$, or from our interpretation of $v^{k i}$, that

$$
\left(v^{k i}\right)^{\prime} x^{*}=0,
$$

implying that inequality (34) binds at the mixed equilibrium $x^{*}$. Moreover, expressions (32) and (33) for $v^{k i}$ and the fact that $A_{k-i}^{k-i}=A_{k-j}^{k-i}+A_{j-i}^{k-i}$ imply that

$$
\begin{aligned}
\left(v^{k i}\right)^{\prime}\left(e_{i}-e_{k}\right) & =\frac{1}{A_{k-i}^{k-i}}\left(A_{k-j}^{k-i} A_{i-k}^{i-k}-A_{k-i}^{k-i} A_{i-k}^{j-k}\right)=Q, \\
\left(v^{k i}\right)^{\prime}\left(e_{k}-e_{j}\right) & =\frac{1}{A_{k-i}^{k-i}}\left(A_{k-j}^{k-i} A_{k-j}^{i}-A_{k-i}^{k-i} A_{k-j}^{j}+A_{i-j}^{i-k} A_{k-j}^{k}\right) \\
& =\frac{1}{A_{k-i}^{k-i}}\left(A_{k-j}^{k-i} A_{j-k}^{j-i}+A_{j-i}^{k-i} A_{j-k}^{j-k}\right)>0, \text { and } \\
\left(v^{k i}\right)^{\prime}\left(e_{i}-e_{j}\right) & =\frac{1}{A_{k-i}^{k-i}}\left(A_{k-j}^{k-i} A_{i-j}^{i-k}+A_{k-i}^{k-i} A_{j-i}^{j-k}\right)>0 .
\end{aligned}
$$

We illustrate the consequences of these relations in Figure 3. Figure 3(i) illustrates inequality (34) when $Q=0$, so that $A$ is a potential game. In this case, equation (36) says that the line on which (34) binds is parallel to $e_{i}-e_{k}$. Thus, by our interpretation of $v^{k i}$, whether mixed strategy $x^{k i}$ or mixed strategy $x^{*}$ is a better response to state $x$ depends only on the value of $x_{j}$. Inequalities (37) and (38) imply that (34) is satisfied at states $e_{i}$ and $e_{k}$, but not at state $e_{j}$, which also agrees with our interpretation of $v^{k i}$.

Figure 3(ii) illustrates inequality (34) when $Q>0$, so that $A$ has clockwise skew. In

\footnotetext{
${ }^{10}$ For motivation, note that $Q=A^{i}\left(e_{j}-e_{k}\right)+A^{j}\left(e_{k}-e_{i}\right)+A^{k}\left(e_{i}-e_{j}\right)$ represents a composite effect on payoffs of a clockwise circuit of the vertices of $X$.
} 


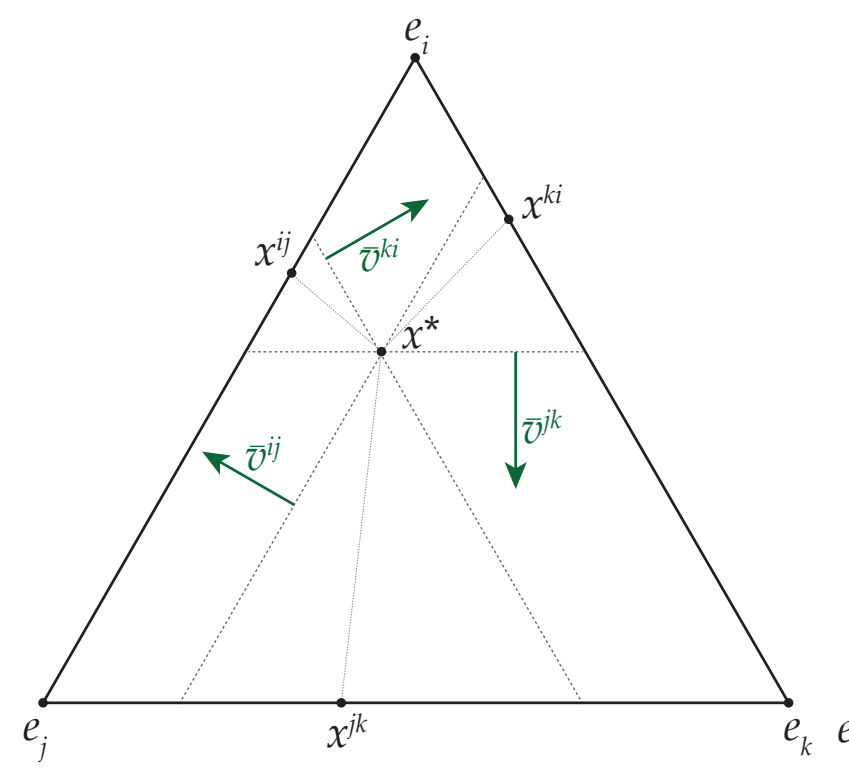

(i) a potential game $(Q=0)$

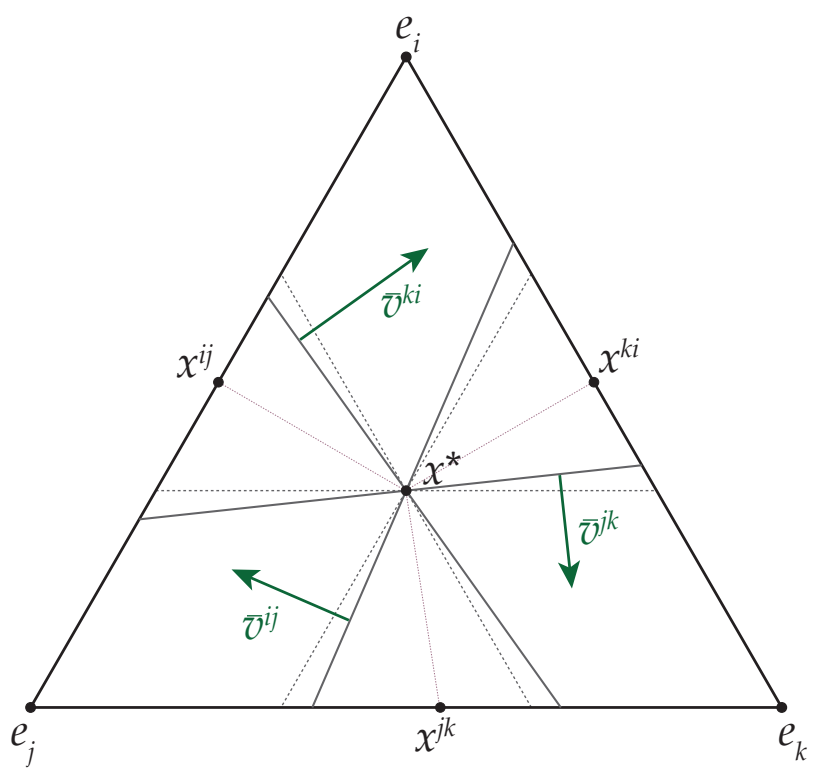

(ii) a clockwise skewed game $(Q>0)$

Figure 3: Skew and inequality (34) in coordination games with the marginal bandwagon property. The vector $\bar{v}^{k i}=\Phi v^{k i}$ is the orthogonal projection of the normal vector $v^{k i}$ onto the tangent space TX.

this case, equation (36) says that the line on which (34) binds is rotated counterclockwise through $x^{*}$ relative to the unskewed case. Inequality (37) implies that this rotation is less than $60^{\circ}$, so that the line where (34) binds passes through the same sextant as mixed equilibrium $x^{i j}$. Finally, inequality (38) implies that (34) is satisfied at state $e_{i}$, but not at state $e_{j} .{ }^{11}$

To complete the initial step of the analysis, let us consider states that are in the region $R^{i k} \subseteq \mathcal{B}^{i}$ introduced above and that are close to $\mathcal{B}^{i k}$. States in the latter set can be expressed as $x^{*}+d \zeta^{k i}$ with $d \geq 0$. Since equation (28) says that the vector $\zeta^{k i}$ is a convex combination of $e_{k}-e_{j}$ and $e_{i}-e_{j}$, equation (35) and inequalities (37) and (38) imply that

$$
\left(v^{k i}\right)^{\prime}\left(x^{*}+d \zeta^{k i}\right)=d\left(v^{k i}\right)^{\prime} \zeta^{k i} \geq 0 .
$$

Thus Lemma 4.1 implies that at states in $R^{i k}$ close to $\mathcal{B}^{i k}$, the value function (30) generated by control $e_{k}-e_{i}$ satisfies the HJB equation (19).

\footnotetext{
${ }^{11}$ If $Q<0$, similar logic shows that the rotation of the line where (34) binds is clockwise relative to the unskewed case, and again less than $60^{\circ}$.
} 


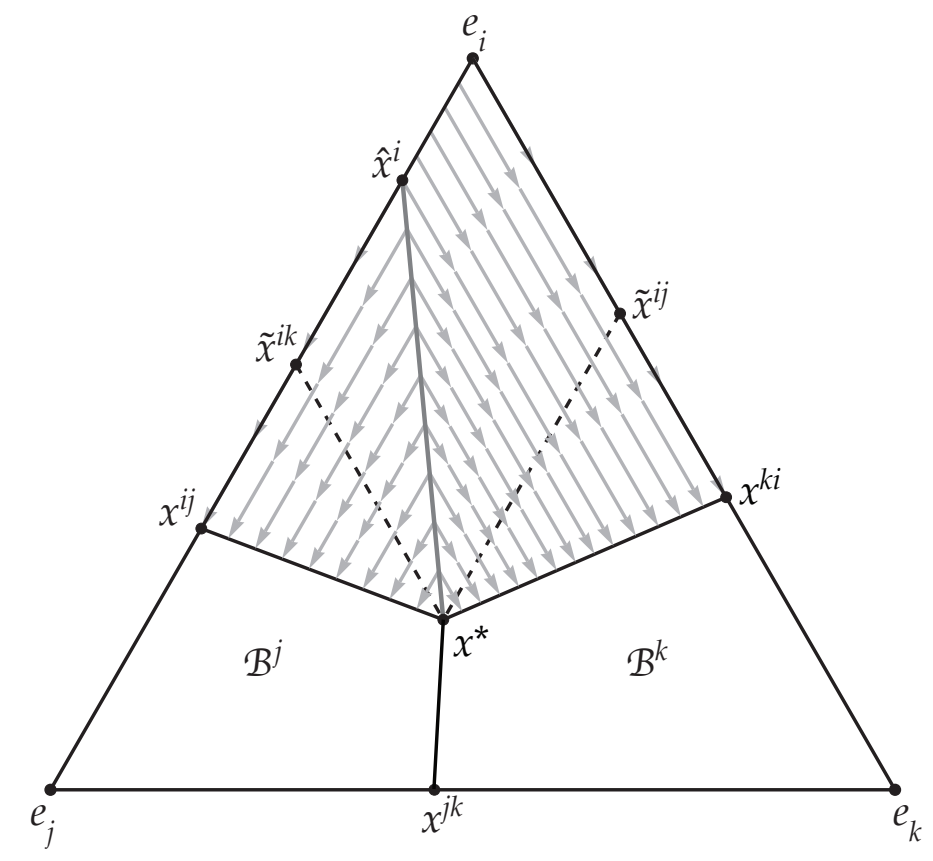

Figure 4: Optimal exit paths from $\mathcal{B}^{i}$ when $\hat{x}^{i}$ is on face $e_{i} e_{j}$.

\section{Characterization of Exit Costs}

We now turn to the exit cost problem (14), whose solutions describe the expected time until the stochastic evolutionary process $\mathbf{X}^{\mathrm{N}, \eta}$ escapes an equilibrium's strong basin of attraction, as well as the likely point of exit.

To begin, we hypothesize that the optimal feedback control takes the form shown in Figure 4. There best response region $\mathcal{B}^{i}$ is split into two regions; in one the optimal control is $e_{j}-e_{i}$, and exit paths lead to $\mathcal{B}^{i j}$; in the other the optimal control is $e_{k}-e_{i}$, and exit paths lead to $\mathcal{B}^{i k}$. The boundary between the regions is a ray whose endpoint is the mixed equilibrium $x^{*}$, and that passes through a state $\hat{x}^{i}$ determined below. From points on this ray, motion in either basic direction is optimal.

State $\hat{x}^{i}$ is identified in Lemma 5.1. To state the lemma, we define

$$
V^{j}(x)=\frac{1}{2} \frac{\left(A^{i-j} x\right)^{2}}{A_{i-j}^{i-j}} \text { and } V^{k}(x)=\frac{1}{2} \frac{\left(A^{i-k} x\right)^{2}}{A_{i-k}^{i-k}} .
$$

By Lemma 3.4, $V^{j}(x)$ is the cost of a path from state $x$ that moves through $\mathcal{B}^{i}$ in direction $e_{j}-e_{i}$ until reaching boundary $\mathcal{B}^{i j} . V^{k}(x)$ is interpreted analogously.

Lemma 5.1. There is a unique state $\hat{x}^{i} \in \mathcal{B}^{i} \cap \mathrm{bd}(X)$ such that 


$$
\begin{aligned}
& \hat{x}_{j}^{i}<x_{j}^{*} \text { and } \hat{x}_{k}^{i}<x_{k^{\prime}}^{*} \\
& \left(v^{i j}\right)^{\prime} \hat{x}^{i}>0 \text { and }\left(v^{i k}\right)^{\prime} \hat{x}^{i}>0, \text { and } \\
& V^{j}\left(\hat{x}^{i}\right)=V^{k}\left(\hat{x}^{i}\right) .
\end{aligned}
$$

Condition (39) of the lemma says that $\hat{x}_{i}$ places less mass on either strategy $j$ or strategy $k$ than the mixed equilibrium $x^{*}$, and so lies above the dashed lines in Figure 4 . This ensures that motion in direction $e_{j}-e_{i}$ from states to the left of the ray through $\hat{x}^{i}$ leads to boundary $\mathcal{B}^{i j}$, and that motion in direction $e_{k}-e_{i}$ from states to the right of the ray leads to $\mathcal{B}^{i k}$. Condition (40) further restricts $\hat{x}^{i}$ to the positive half-spaces defined by normal vectors $v^{i j}$ and $v^{i k}$ (see Figure 3). By Lemma 4.1, these conditions ensure that $V^{j}$ satisfies the HJB equation (19) to the left of the ray through $\hat{x}^{i}$, and that $V^{k}$ satisfies (19) to the right of the ray. Finally, condition (41) says that from state $\hat{x}^{i}$, the costs of proceeding in direction $e_{j}-e_{i}$ to $\mathcal{B}^{i j}$ and in direction $e_{k}-e_{i}$ to $\mathcal{B}^{i k}$ are equal. Since $V^{j}(x)$ and $V^{k}(x)$ are homogenous of degree 2 in the displacement $z=x-x^{*}$ from $x^{*}$, condition (41) ensures that $V^{j}$ and $V^{k}$ match up continuously along the ray from $x^{*}$ through $\hat{x}_{i}$, as required by the verification theorem.

This last argument illustrates an important general point. Throughout the paper, the linear inequalities we use to define control regions bind at $x^{*}$; so do the linear inequalities from Lemma 3.2 used to check the HJB equation. Consequently, our construction of the value function and our arguments to check the HJB equation apply throughout the affine space $\operatorname{aff}(X)$, as required by Theorem 3.1 .

The proof of Lemma 5.1 is presented in Appendix A.2. In brief, the proof considers the behavior of the difference $V^{k}-V^{j}$ on the lines $\ell^{i j}=\left\{s e_{i}+(1-s) e_{j}: s \in \mathbb{R}\right\}$ and $\ell^{i k}=\left\{s e_{i}+(1-s) e_{k}: s \in \mathbb{R}\right\}$ through aff $(X)$ (see Figure 9 in Appendix A.2). It is easy to check that $V^{k}-V^{j}$ is quadratic on each of these lines, and that it is concave on $\ell^{i j}$ and convex on $\ell^{i k}$. Computations show that $V^{k}-V^{j}$ admits two zeros on each line; the zeros of interest, denoted $y^{i j}$ and $y^{i k}$, are those with the larger $i$ components. By definition, these points satisfy condition (41), and further computations confirm that they satisfy conditions (39) and (40), and that $y^{i j}, y^{i k}$, and $x^{*}$ are collinear. If $y^{i j}$ and $y^{i k}$ are both $e_{i}$, we set $\hat{x}^{i}=e_{i}$. If not, exactly one of $y^{i j}$ and $y^{i k}$ is in $X$, and that one is our $\hat{x}^{i}$.

With Lemma 5.1 in hand, we can describe the value function and the optimal feedback control for the exit problem. To do so, we define the cross product

$$
w^{i}=x^{*} \times\left(\hat{x}^{i}-x^{*}\right)
$$

to be a vector normal to segment $\hat{x}^{i} x^{*}$. By the right-hand rule (see Section 4.3), states 
satisfying $\left(w^{i}\right)^{\prime} x>0$ appear to the left of segment $\hat{x}^{i} x^{*}$ in Figure 4 . It is convenient to focus on controls from the set

$$
X \ominus X=\{\alpha-\beta: \alpha, \beta \in X, \operatorname{supp}(\alpha) \cap \operatorname{supp}(\beta)=\varnothing\}=\operatorname{bd}(Z),
$$

since every nonzero element of $Z$ is proportional to an element of this set. For concision, the results to come do not say explicitly that the value function equals zero on the target sets, nor do they specify that the optimal control on those sets is the null control.

Proposition 5.2. If $A$ is a simple three-strategy coordination game, the value function $V^{*}: \mathcal{B}^{i} \rightarrow$ $\mathbb{R}_{+}$for the exit cost problem (14) with target set $\mathcal{B}^{j} \cup \mathcal{B}^{k}$ is the continuous function

$$
V^{*}(x)= \begin{cases}\frac{1}{2} \frac{\left(A^{i-k} x\right)^{2}}{A_{i-k}^{i-k}} & \text { if }\left(w^{i}\right)^{\prime} x \leq 0, \\ \frac{1}{2} \frac{\left(A^{i-j} x\right)^{2}}{A_{i-j}^{i-j}} & \text { if }\left(w^{i}\right)^{\prime} x>0 .\end{cases}
$$

The optimal feedback controls with range $X \ominus X$ are

$$
v^{*}(x) \begin{cases}=e_{k}-e_{i} & \text { if }\left(w^{i}\right)^{\prime} x<0 \\ \in\left\{e_{k}-e_{i}, e_{j}-e_{i}\right\} & \text { if }\left(w^{i}\right)^{\prime} x=0, \\ =e_{j}-e_{i} & \text { if }\left(w^{i}\right)^{\prime} x>0 .\end{cases}
$$

Proof. We apply the verification theorem. The value function $V^{*}$ in (42) is constructed from feedback controls (43) that generate feasible solutions to the exit problem, as required by condition (i) of Theorem 3.1. The continuity of $V$ follows from Lemma 5.1 and the argument in the subsequent paragraph. $V^{*}$ is clearly $C^{1}$ off the set $\left\{x \in \operatorname{aff}(X):\left(w^{i}\right)^{\prime} x=0\right\}$, and Lemmas 4.1 and 5.1 imply that the HJB equation holds away from this set. Thus condition (ii) of Theorem 3.1 is satisfied, and the proof is complete.

Proposition 5.2 yields the solution to exit problem (14):

Corollary 5.3. In a simple three-strategy coordination game,

$$
C\left(\left\{e_{i}\right\}, \mathcal{B}^{j} \cup \mathcal{B}^{k}\right)=\min \left\{\gamma\left(e_{i}, x^{i j}\right), \gamma\left(e_{i}, x^{k i}\right)\right\}=\min \left(\frac{1}{2} \frac{\left(A^{i-j} e_{i}\right)^{2}}{A_{i-j}^{i-j}}, \frac{1}{2} \frac{\left(A^{i-k} e_{i}\right)^{2}}{A_{i-k}^{i-k}}\right) .
$$




\section{Characterization of Transition Costs}

In this section, we consider the transition cost problem (15), whose solutions are used to describe the global long-run behavior of the process $\mathbf{X}^{N, \eta}$.

Unlike that of exit costs, the analysis of transition costs depends in a basic way on whether the game at hand is a potential game. To see why, we recall the reasoning from Section 4.2, where we sought to define a region in $\mathcal{B}^{i}$ from which optimal paths to $\mathcal{B}^{k}$ proceed in direction $e_{k}-e_{i}$ to $\mathcal{B}^{i k}$, generating value function (30) in that region. By Lemma 4.1, this value function is consistent with the HJB equation (19) whenever $\left(v^{k i}\right)^{\prime} x \geq 0$.

Suppose first that $A$ is a potential game, so that the skew $Q$ equals zero. In this case, Figure 3(i) shows that states in $\mathcal{B}^{i}$ satisfying $x_{j} \leq x_{j}^{*}$, from which motion in direction $e_{k}-e_{i}$ leads to $\mathcal{B}^{k i}$, also satisfy inequality (34). It is therefore consistent with the analysis so far for optimal paths to proceed in direction $e_{k}-e_{i}$ to $\mathcal{B}^{k}$ whenever feasible.

If instead $A$ has clockwise skew, so that $Q>0$, Figure 3(ii) shows that the same conclusion about motion from $\mathcal{B}^{i}$ to $\mathcal{B}^{k}$ obtains. However, we cannot reach the analogous conclusion about motion from $\mathcal{B}^{j}$ to $\mathcal{B}^{k}$. In the thin triangle to the left of $x^{*}$, motion in direction $e_{k}-e_{j}$ leads to $\mathcal{B}^{j k}$. But since $\left(v^{j k}\right)^{\prime} x<0$ here, this motion is not consistent with the HJB equation (19). Thus the optimal paths to $\mathcal{B}^{k}$ must take a different form, a form we determine in Section 6.2.

\subsection{Transition costs in potential games}

In potential games, the value function for the transition cost problem (15) is easy to describe, and is even smooth, but the optimal feedback controls are of a degenerate form.

Proposition 6.1. Let $A$ be a simple three-strategy coordination game, and suppose that $A$ is a potential game. Then the value function $V^{*}: \mathcal{B}^{i} \cup \mathcal{B}^{j} \rightarrow \mathbb{R}_{+}$for the transition cost problem (15) with target set $\mathcal{B}^{k}$ is the $C^{1}$ function

$$
V^{*}(x)= \begin{cases}\frac{1}{2} \frac{\left(A^{i-k} x\right)^{2}}{A_{i-k}^{i-k}} & \text { if } x_{j}<x_{j}^{*}, \\ \frac{1}{2}\left(x-x^{*}\right)^{\prime} A\left(x-x^{*}\right) & \text { if } x_{j} \geq x_{j}^{*} \text { and } x_{i} \geq x_{i}^{*}, \\ \frac{1}{2} \frac{\left(A^{j-k} x\right)^{2}}{A_{j-k}^{j-k}} & \text { if } x_{i}<x_{i}^{*} .\end{cases}
$$

The optimal feedback controls with range $X \ominus X$ are 


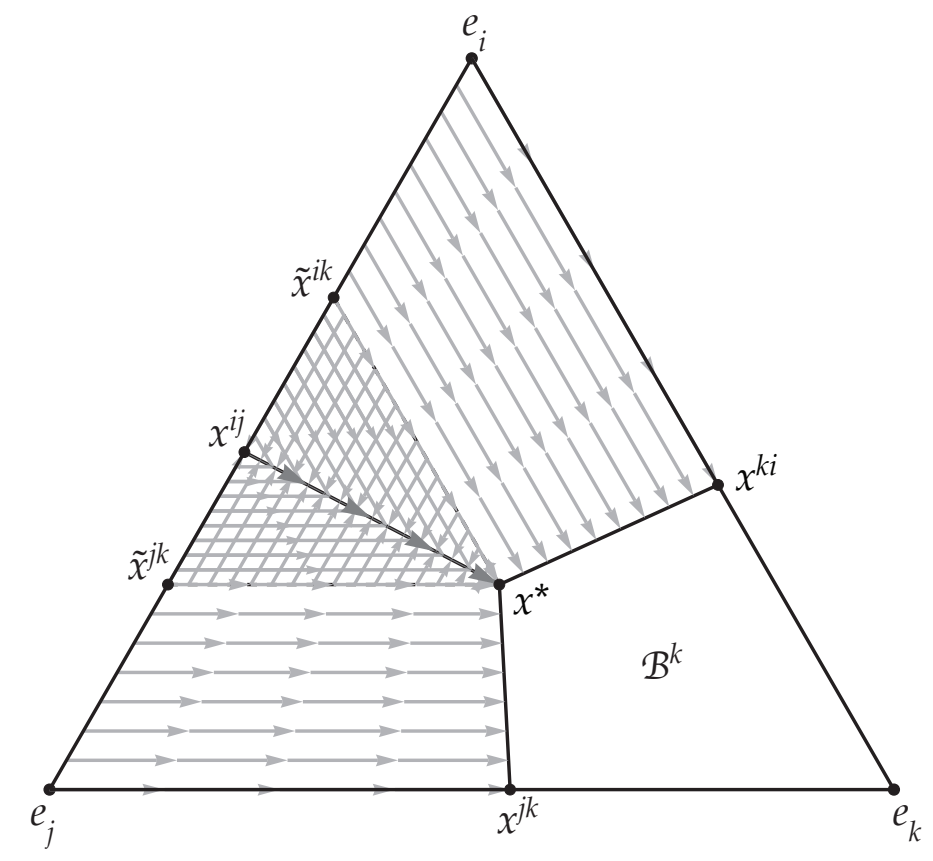

Figure 5: Optimal transition paths to $\mathcal{B}^{k}$ in a potential game $(Q=0)$. In the crosshatched regions, continuous sets of control directions are optimal.

$$
v^{*}(x) \begin{cases}=e_{k}-e_{i} & \text { if } x_{j}<x_{j}^{*}, \\ \in \operatorname{conv}\left(\left\{e_{j}-e_{i}, e_{k}-e_{i}\right\}\right) & \text { if } x_{j} \geq x_{j}^{*} \text { and } A^{i-j} x>0, \\ =-\zeta^{i j} & \text { if } A^{i-j} x=0, \\ \in \operatorname{conv}\left(\left\{e_{i}-e_{j}, e_{k}-e_{j}\right\}\right) & \text { if } x_{i} \geq x_{i}^{*} \text { and } A^{i-j} x<0, \\ =e_{k}-e_{j} & \text { if } x_{i}<x_{i}^{*} .\end{cases}
$$

The feedback controls (45) are illustrated in Figure 5. At states in the sextant northwest of $x^{*}$ other than those on the ray through $x^{i j}$, continuous ranges of control vectors are optimal. This degeneracy is particular to potential games, as we explain below.

Proof of Proposition 6.1. To apply the verification theorem, we first show that $V^{*}$ is $C^{1}$. This is clearly true inside each of the three regions in the piecewise definition (44). It remains to consider the behavior of $V^{*}$ at states satisfying $x_{j}=x_{j}^{*}$ or $x_{i}=x_{i}^{*}$. We focus on the former states. Such states satisfy $x=x^{*}+d\left(e_{k}-e_{i}\right)$ for some $d \geq 0$. It follows that $V^{*}$ is continuous at such states, since

$$
V^{1}(x) \equiv \frac{1}{2} \frac{\left(A^{i-k} x\right)^{2}}{A_{i-k}^{i-k}}=\frac{1}{2} d^{2} A_{i-k}^{i-k}=\frac{1}{2}\left(x-x^{*}\right)^{\prime} A\left(x-x^{*}\right) \equiv V^{2}(x) .
$$

To check that $V^{*}$ is $C^{1}$, recall from Section 2.2 that since $A$ is a potential game, we can write 
$A=C+\mathbf{1} r^{\prime}$ for some symmetric matrix $C \in \mathbb{R}^{n \times n}$ and some vector $r \in \mathbb{R}^{n}$. Using these facts and the fact that $x^{*}$ is an interior Nash equilibrium of both $A$ and $C$, we have

$$
\begin{aligned}
V^{2}(x) & =\frac{1}{2}\left(x-x^{*}\right)^{\prime} A\left(x-x^{*}\right)=\frac{1}{2}\left(x-x^{*}\right)^{\prime} C\left(x-x^{*}\right)=\frac{1}{2}\left(x-x^{*}\right)^{\prime} C x \\
& =\frac{1}{2}\left(x^{\prime} C x-x^{\prime} C x^{*}\right)=\frac{1}{2}\left(x^{\prime} C x-\left(x^{*}\right)^{\prime} C x^{*}\right) .
\end{aligned}
$$

Thus for $z \in T X$, these facts and the symmetry of $A$ with respect to $T X \times T X$ yield

$$
D V^{2}(x) z=x^{\prime} C z=z^{\prime} C x=z^{\prime} A x=z^{\prime} A\left(x-x^{*}\right)=\left(x-x^{*}\right)^{\prime} A z .
$$

But at states $x$ with $x_{j}=x_{j}^{*}$, the fact that $A^{i-k} x^{*}=0$ and the definition of $d$ imply that

$$
D V^{1}(x) z=\frac{A^{i-k} x}{A_{i-k}^{i-k}} A^{i-k} z=\frac{A^{i-k}\left(x-x^{*}\right)}{A_{i-k}^{i-k}} A^{i-k} z=d A^{k-i} z=\left(x-x^{*}\right)^{\prime} A z,
$$

so $V^{*}$ is $C^{1}$ at these states.

Next, we show that the value function $V^{*}$ is generated by the controls (45). For the first and third cases of definition (44), this follows from Lemma 3.4. To address the second case, we require the following lemma, which applies equally well to the other cases (see the discussion following this proof). The lemma uses the fact that the function $f: \operatorname{aff}(X) \rightarrow \mathbb{R}$ defined by $f(x)=\frac{1}{2} x^{\prime} C x$ is a potential function for $F(x)=A x$ on aff $(X)$, in the sense that $D f(x) z=F(x)^{\prime} z=z^{\prime} A x$ for all $z \in T X$ and $x \in \operatorname{aff}(X) .^{12}$

Lemma 6.2. The cost $c(\phi)$ of trajectory $\phi:[0, T] \rightarrow$ aff $(X)$ satisfies $c(\phi) \geq f\left(\phi_{0}\right)-f\left(\phi_{T}\right)$. If for each $t \in(0, T)$, every strategy $h$ with $\left(\dot{\phi}_{t}\right)_{h}<0$ is optimal at $\phi_{t}$, then $c(\phi)=f\left(\phi_{0}\right)-f\left(\phi_{T}\right)$.

Proof. By definition (13) of path costs, and since $\left[\dot{\phi}_{t}\right]_{+}^{\prime} \mathbf{1}=\left[\dot{\phi}_{t}\right]_{-}^{\prime} \mathbf{1}$ and $\mathbf{1} A^{\breve{b}\left(\phi_{t}\right)} \phi_{t} \geq A \phi_{t}$,

$$
\begin{aligned}
c(\phi) & =\int_{0}^{T}\left[\dot{\phi}_{t}\right]_{+}^{\prime}\left(1 A^{\check{b}\left(\phi_{t}\right)}-A\right) \phi_{t} \mathrm{~d} t \geq \int_{0}^{T}\left(\left[\dot{\phi}_{t}\right]_{-}^{\prime} A-\left[\dot{\phi}_{t}\right]_{+}^{\prime} A\right) \phi_{t} \mathrm{~d} t \\
& =-\int_{0}^{T} \dot{\phi}_{t}^{\prime} A \phi_{t} \mathrm{~d} t=-\int_{0}^{T} D f\left(\phi_{t}\right) \dot{\phi}_{t} \mathrm{~d} t=f\left(\phi_{0}\right)-f\left(\phi_{T}\right) .
\end{aligned}
$$

If the assumption on the support of $\left[\dot{\phi}_{t}\right]_{-}$holds, then $\left[\dot{\phi}_{t}\right]_{-}^{\prime} \mathbf{1} A^{\check{b}\left(\phi_{t}\right)} \phi_{t}=\left[\dot{\phi}_{t}\right]_{-}^{\prime} A \phi_{t}$, so the inequality in the display binds.

Proceeding with our earlier argument, we note that any controlled path $\phi:[0, T] \rightarrow$ aff $(X)$ starting from a state $x$ with $x_{j} \geq x_{j}^{*}$ and $x_{i} \geq x_{i}^{*}$ and generated by controls satisfying

\footnotetext{
${ }^{12}$ See Hofbauer and Sigmund (1988) and Sandholm (2001, 2009).
} 
(45) both satisfies the assumption of Lemma 6.2 and terminates at $\phi_{T}=x^{*}$ (see Figure 5). Thus Lemma 6.2, the definition of $f$, and equation (46) yield

$$
c(\phi)=f(x)-f\left(x^{*}\right)=\frac{1}{2} x^{\prime} C x-\frac{1}{2}\left(x^{*}\right)^{\prime} C x^{*}=\frac{1}{2}\left(x-x^{*}\right)^{\prime} A\left(x-x^{*}\right),
$$

as specified in the second case of (44).

The proposition will follow from Theorem 3.1 if we can show that $V^{*}$ satisfies the HJB equation (19) at all states. Since $A$ is a potential game, the states with $\left(v^{k i}\right)^{\prime} x>0$ are those satisfying $x_{j}<x_{j}^{*}$ (see Figure 3(i)), so Lemma 4.1 implies $V^{*}$ satisfies (19) at these states. Analogous reasoning shows that $V^{*}$ satisfies (19) when $x_{i}<x_{i}^{*}$. It thus remains to check (19) at states satisfying $x_{j} \geq x_{j}^{*}$ and $x_{i} \geq x_{i}^{*}$. To do so, we show that (19) holds when $x_{j} \geq x_{j}^{*}$ and $A^{i-j} x>0$; the argument when $x_{i} \geq x_{i}^{*}$ and $A^{i-j} x<0$ is similar; and then (19) must hold when $x_{j}=x_{j}^{*}, x_{i}=x_{i}^{*}$, or $A^{i-j} x=0$ by virtue of the fact that $V^{*}$ is $C^{1}$.

So suppose that $x$ satisfies $x_{j} \geq x_{j}^{*}$ and $A^{i-j} x>0$. Since $D V^{*}(x) z=z^{\prime} A x$ at such states by equation (47), substitution into the HJB equation (19) yields

$$
\min _{e_{a}, e_{b} \neq e_{a}}\left(e_{i}-e_{b}\right)^{\prime} A x=0 .
$$

Since $x$ is in $\mathcal{B}^{i}$ but not in $\mathcal{B}^{j}$ or $\mathcal{B}^{k}$ (see Figure 5), minimization in (48) requires setting $e_{b}=e_{i}$. Then choosing $e_{a}$ to be either $e_{j}$ or $e_{k}$ attains the minimum of $0 .{ }^{13}$ This completes the proof of the proposition.

Because the integrand of the cost function (13) is piecewise linear in the control $u=\dot{\phi}_{t}$, it is natural to expect the optimal control in $X \ominus X$ to be unique at almost all states. That this is not true here is a consequence of the integrability properties that define potential games, a point we now consider from two points of view.

First, along any controlled trajectory generated by (45), agents only switch from optimal strategies to suboptimal strategies. Thus Lemma 6.2 implies that for every state $x, V^{*}(x)$ is equal to the change in potential between state $x$ and the terminal state of the controlled trajectory. When there are multiple controlled trajectories between the initial and terminal states, as in the second and fourth cases of (45), each achieves this same minimal cost. ${ }^{14}$

\footnotetext{
${ }^{13}$ To consider all controls in $X \ominus X$, we must write the HJB equation in form (18); then the previous argument and the piecewise linearity of (18) implies that the set of optimal controls is conv $\left(\left\{e_{j}-e_{i}, e_{k}-e_{i}\right\}\right)$, as described in the second case of (45).

${ }^{14}$ To address a possible misconception, let us consider an initial state $x=(1-c) e_{i}+c e_{j}$ with $c \in\left(0, x_{j}^{*}\right)$. Proposition 6.1 says that the optimal path from $x$ to $\mathcal{B}^{k}$ proceeds in direction $e_{k}-e_{i}$ until reaching the state $y \in \mathcal{B}^{i k}$ with $y_{j}=c$. The argument above shows that this path's cost is $f(x)-f(y)$. One might wonder why there is not a lower cost path that terminates at the mixed equilibrium $x^{k i}$ : since $f\left(x^{k i}\right)$ is greater than $f(y)$, $f(x)-f\left(x^{k i}\right)$ is less than $f(x)-f(y)$. But along any path from $x$ that first hits $\mathcal{B}^{i k}$ at $x^{k i}$, some agents must
} 
Second, we argue that $A$ being a potential game is a necessary condition for having a region in $\mathcal{B}^{i}$ where both $e_{j}-e_{i}$ and $e_{k}-e_{i}$ are optimal controls. Equation (22) implies that in the interior of such a region, the value function must satisfy

$$
D V(x)\left(e_{j}-e_{i}\right)=A^{j-i} x \text { and } D V(x)\left(e_{k}-e_{i}\right)=A^{k-i} x \text {. }
$$

Since $e_{j}-e_{i}$ and $e_{k}-e_{i}$ span $T X$, these equalities imply that

$$
D V(x) z=z^{\prime} A x \text { for all } z \in T X \text {. }
$$

Thus the second derivative $D^{2} V(x)$ is given by

$$
D^{2} V(x)(z, \hat{z})=z^{\prime} A \hat{z} \text { for all } z, \hat{z} \in T X
$$

The first expression is symmetric in $z$ and $\hat{z}$, by virtue of being a second derivative. Thus $A$ is symmetric with respect to $T X \times T X$, and so is a potential game.

Proposition 6.1 yields the solution to the transition cost problem (15) in potential games.

Corollary 6.3. If the simple three-strategy coordination game $A=C+1 r^{\prime}$ is a potential game, so that $f(x)=\frac{1}{2} x^{\prime} C x$ is a potential function for $F(x)=A x$, then

$$
C\left(\left\{e_{i}\right\}, \mathcal{B}^{k}\right)=\gamma\left(e_{i}, x^{k i}\right)=\frac{1}{2} \frac{\left(A^{i-k} e_{i}\right)^{2}}{A_{i-k}^{i-k}}=f\left(e_{i}\right)-f\left(x^{k i}\right) .
$$

\subsection{Transition costs in skewed games}

We now consider the transition cost problem (15) in games with clockwise skew: $Q>0$.

It is natural to expect that if the skew $Q$ is small, then the optimal control should resemble the one from the $Q=0$ case from Figure 5. The previous discussion shows that once $Q$ is positive, no region will have multiple optimal controls. Thus the form of the control in the sextant northwest of $x^{*}$ must change.

At the start of this section, we argued that in clockwise-skewed games, motion from $\mathcal{B}^{i}$ to $\mathcal{B}^{k i}$ in direction $e_{k}-e_{i}$ is consistent with the HJB equation whenever such a path exists. We therefore hypothesize that motion is in direction $e_{k}-e_{i}$ throughout the interior of $\mathcal{B}^{i}$, even when such motion leads to boundary $\mathcal{B}^{i j}$. We also saw that motion from $\mathcal{B}^{j}$ to $\mathcal{B}^{j k}$ in direction $e_{k}-e_{j}$ is not always consistent with the HJB equation. This leads us to

abandon the suboptimal strategy $j$. Thus Lemma 6.2 does not apply, and indeed, the cost of such a path exceeds $f(x)-f\left(x^{k i}\right)$. The cheapest path from $x$ to $x^{k i}$ goes first from $x$ to $y$ at cost $f(x)-f(y)$, and then from $y$ to $x^{i j}$ at zero cost. Proposition 6.1 implies that no path can reach $x^{i j}$ more cheaply. 
hypothesize that in a portion of $\mathcal{B}^{j}$ close to $\mathcal{B}^{i j}$, motion will instead be in direction $e_{i}-e_{j}$.

The conjectured form of the optimal control is pictured in Figures 6 and 7. In the sextant northwest of $x^{*}$, the multiple optimal controls from Figure 5 have been replaced in Figures 6 and 7 with selections from these controls. The boundary $\mathcal{B}^{i j}$ is approached from states on both sides, but it is approached obliquely from the $\mathcal{B}^{i}$ side, and nearly squarely from the $\mathcal{B}^{j}$ side. In the remainder of this section, we prove that that the optimal value function is generated by feedback controls of the form shown in these figures.

To begin, we introduce notation for the endpoints of paths that proceed in a basic direction until reaching a boundary between best response regions. Using Lemma 3.4, and proceeding from top to bottom in Figure 6 or 7, we have

$$
\begin{aligned}
& \text { for } x \in \mathcal{B}^{i} \text { with } x_{j} \leq x_{j}^{*} \text {, let } \omega^{i k}(x)=x+\left(e_{k}-e_{i}\right) d^{i k}(x) \in \mathcal{B}^{k i} \text {, where } d^{i k}(x)=\frac{A^{i-k} x}{A_{i-k}^{i-k}}, \\
& \text { for } x \in \mathcal{B}^{i} \text { with } x_{j} \geq x_{j}^{*} \text {, let } \chi^{i k}(x)=x+\left(e_{k}-e_{i}\right) \delta^{i k}(x) \in \mathcal{B}^{i j} \text {, where } \delta^{i k}(x)=\frac{A^{i-j} x}{A_{i-k}^{i-j}}, \\
& \text { for } x \in \mathcal{B}^{j} \text { with } x_{k} \leq x_{k}^{*} \text {, let } \chi^{j i}(x)=x+\left(e_{i}-e_{j}\right) d^{j i}(x) \in \mathcal{B}^{i j} \text {, where } d^{j i}(x)=\frac{A^{j-i} x}{A_{j-i}^{j-i}}, \\
& \text { for } x \in \mathcal{B}^{j} \text { with } x_{i} \leq x_{i}^{*} \text {, let } \omega^{j k}(x)=x+\left(e_{k}-e_{j}\right) d^{j k}(x) \in \mathcal{B}^{j k} \text {, where } d^{j k}(x)=\frac{A^{j-k} x}{A_{j-k}^{j-k}} .
\end{aligned}
$$

Using these definitions, we can define the pieces of the value function. Again proceeding from top to bottom, we have

$$
\begin{aligned}
& V^{1}(x)=\gamma\left(x, \omega^{i k}(x)\right), \\
& V^{2}(x)=\gamma\left(x, \chi^{i k}(x)\right)+\gamma\left(\chi^{i k}(x), x^{*}\right), \\
& V^{3}(x)=\gamma\left(x, \chi^{j i}(x)\right)+\gamma\left(\chi^{j i}(x), x^{*}\right), \\
& V^{4}(x)=\gamma\left(x, \omega^{j k}(x)\right) .
\end{aligned}
$$

Looking at Figures 6 and 7, the only characteristic of the proposed value function that remains ambiguous is the boundary between the regions where definitions $V^{3}$ and $V^{4}$ apply. In the figure, this boundary is a ray from mixed equilibrium $x^{*}$ through a state $\hat{x}^{j k}$ on the boundary of $X$. This state is identified in the following lemma.

Lemma 6.4. If $A$ has clockwise skew, then there is a unique state $\hat{x}^{j k} \in \mathcal{B}^{j} \cap \mathrm{bd}(X)$ such that

$$
\hat{x}_{k}^{j k}<x_{k}^{*}
$$




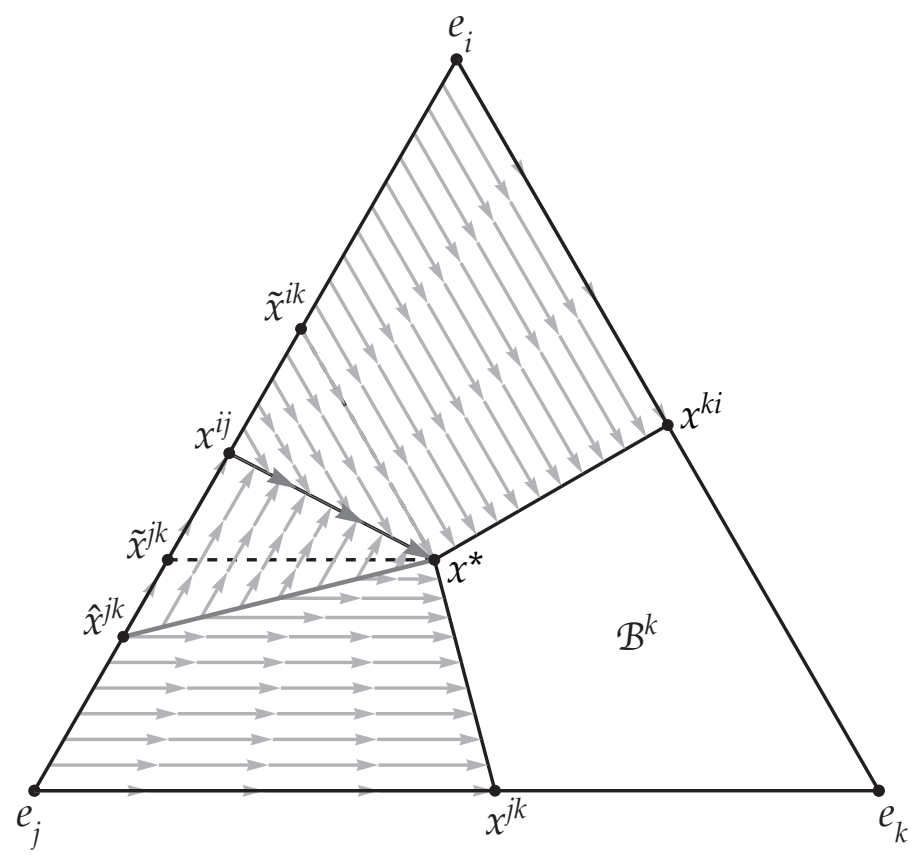

Figure 6: Optimal transition paths to $\mathcal{B}^{k}$ in a clockwise skewed game when $\hat{x}^{j k}$ is on face $e_{i} e_{j}$.

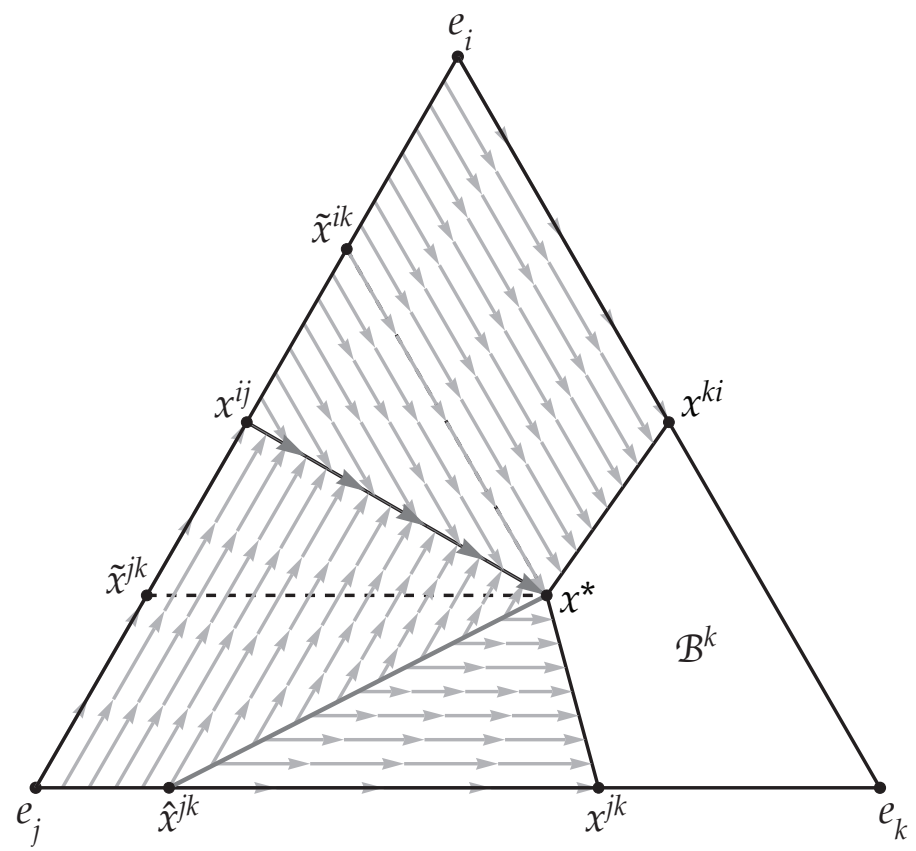

Figure 7: Optimal transition paths to $\mathcal{B}^{k}$ in a clockwise skewed game when $\hat{x}^{j k}$ is on face $e_{j} e_{k}$. 


$$
\begin{aligned}
& \left(v^{j k}\right)^{\prime} \hat{x}^{j k}>0, \text { and } \\
& V^{3}\left(\hat{x}^{j k}\right)=V^{4}\left(\hat{x}^{j k}\right) .
\end{aligned}
$$

The requirement that $\hat{x}^{j k} \in \mathcal{B}^{j} \cap \operatorname{bd}(X)$ means that $\hat{x}^{j k}$ lies on the southwest portion of $\mathrm{bd}(X)$, between mixed equilibria $x^{i j}$ and $x^{j k}$. Condition (49) says that $\hat{x}^{j k}$ lies to the left of the line on which $x_{k}=x_{k}^{*}$, so that motion from $\hat{x}^{j k}$ in direction $e_{i}-e_{j}$ leads to $\mathcal{B}^{i j}$ (rather than $\left.\mathcal{B}^{j k}\right)$. Condition (50) says that $\hat{x}^{j k}$ is below the line where $\left(v^{j k}\right)^{\prime} x=0$. Since $Q$ is positive, it follows that $\hat{x}_{i}^{j k}<x_{i}^{*}$ (see Figure 3(ii)), and thus that motion from $\hat{x}^{j k}$ in direction $e_{k}-e_{j}$ leads to $\mathcal{B}^{j k}$. Finally, condition (51) says that the same cost accrues along the two paths from $\hat{x}^{j k}$ to $\mathcal{B}^{k}$ indicated in Figure 6: the concatenation of the linear segment from $\hat{x}^{j k}$ in direction $e_{i}-e_{j}$ to $\chi^{j i}(x) \in \mathcal{B}^{i j}$ and the segment from $\chi^{j i}(x)$ to $x^{*}$, whose total cost is $V^{3}\left(\hat{x}^{j k}\right)$, and the direct path from $\hat{x}^{j k}$ in direction $e_{k}-e_{j}$ to $\omega^{j k}(x) \in \mathcal{B}^{j k}$, whose cost is $V^{4}\left(\hat{x}^{j k}\right)$. The proof of Lemma 6.4 follows lines similar to the proof of Lemma 5.1, and is presented in Appendix A.3.2.

To state the main result of this section, we define the vector $w^{j k}$ to be the cross product

$$
w^{j k}=x^{*} \times\left(\hat{x}^{j k}-x^{*}\right) .
$$

In Figures 6 and 7, the states satisfying $\left(w^{j k}\right)^{\prime} x>0$ are those below the ray from $x^{*}$ through $\hat{x}^{j k}$.

Proposition 6.5. Let $A$ be a simple three-strategy coordination game with clockwise skew. Then the value function $V^{*}: \mathcal{B}^{i} \cup \mathcal{B}^{j} \rightarrow \mathbb{R}_{+}$for the transition cost problem (15) with target set $\mathcal{B}^{k}$ is

$$
V^{*}(x)= \begin{cases}\gamma\left(x, \omega^{i k}(x)\right) & \text { if } x_{j} \leq x_{j}^{*} \\ \gamma\left(x, \chi^{i k}(x)\right)+\gamma\left(\chi^{i k}(x), x^{*}\right) & \text { if } x_{j}>x_{j}^{*} \text { and } A^{i-j} x \geq 0, \\ \gamma\left(x, \chi^{j i}(x)\right)+\gamma\left(\chi^{j i}(x), x^{*}\right) & \text { if } A^{i-j} x<0 \text { and }\left(w^{j k}\right)^{\prime} x<0, \\ \gamma\left(x, \omega^{j k}(x)\right) & \text { if }\left(w^{j k}\right)^{\prime} x \geq 0,\end{cases}
$$

The optimal feedback controls with range $X \ominus X$ are

$$
v^{*}(x) \begin{cases}=e_{k}-e_{i} & \text { if } A^{i-j} x>0, \\ =-\zeta^{i j} & \text { if } A^{i-j} x=0, \\ =e_{i}-e_{j} & \text { if } A^{i-j} x<0 \text { and }\left(w^{j k}\right)^{\prime} x<0, \\ \in\left\{e_{i}-e_{j}, e_{k}-e_{j}\right\} & \text { if }\left(w^{j k}\right)^{\prime} x=0, \\ =e_{k}-e_{j} & \text { if }\left(w^{j k}\right)^{\prime} x>0 .\end{cases}
$$


In Appendix A.3, we prove Proposition 6.5 by establishing that the value function defined in (52) satisfies the conditions of the verification theorem. After some preliminary calculations (Appendix A.3.1) and the proof of Lemma 6.4 (Appendix A.3.2), we show in Appendix A.3.3 that $V$ is continuous, and that it is differentiable except at states $x$ at which $\left(w^{j k}\right)^{\prime} x=0$. In Appendix A.3.4, we use Lemmas 3.2, 4.1, and 6.4 to show that the HJB equation holds at all other states. The proposition then follows from Theorem 3.1.

Proposition 6.5 implies that in clockwise-skewed games, the possible optimal transition paths depend on the ordering of the strategy pair in question. For a clockwise transition, from $e_{i}$ to $\mathcal{B}_{k}$, the optimal path is always the direct boundary path to $x^{k i}$. For a counterclockwise transition, from $e_{j}$ to $\mathcal{B}_{k}$, the optimal path is either the direct boundary path to $x^{j k}$ (Figure 6), or a two-segment path that proceeds first to mixed equilibrium $x^{i j}$, and from there to interior equilibrium $x^{*}$ (Figure 7). To summarize:

Corollary 6.6. In a simple three-strategy coordination game with clockwise skew,

$$
\begin{aligned}
C\left(\left\{e_{i}\right\}, \mathcal{B}^{k}\right) & =\gamma\left(e_{i}, x^{k i}\right)=\frac{1}{2} \frac{\left(A^{i-k} e_{i}\right)^{2}}{A_{i-k}^{i-k}} \text { and } \\
C\left(\left\{e_{j}\right\}, \mathcal{B}^{k}\right) & =\min \left\{\gamma\left(e_{j}, x^{j k}\right), \gamma\left(e_{j}, x^{i j}\right)+\gamma\left(x^{i j}, x^{*}\right)\right\} \\
& =\min \left\{\frac{1}{2} \frac{\left(A^{j-k} e_{j}\right)^{2}}{A_{j-k}^{j-k}}, \frac{1}{2} \frac{\left(A^{j-i} e_{i}\right)^{2}}{A_{j-i}^{j-i}}+\frac{1}{2}\left(x_{k}^{*}\right)^{2}\left(\zeta^{i j}\right)^{\prime} A \zeta^{i j}\right\} .
\end{aligned}
$$

Remark 6.7. It is worth comparing the exit and transition costs for simple three-strategy coordination games under the logit protocol to those under the BRM protocol of Kandori et al. (1993), in which any switch to a suboptimal strategy has unlikelihood 1. Under the latter, the least cost exit path from $e_{i}$ to $\mathcal{B}^{j} \cup \mathcal{B}^{k}$ follows a boundary to either mixed equilibrium $x^{i j}$ or mixed equilibrium $x^{k i}$, since these are the states in $\mathcal{B}^{i j}$ and $\mathcal{B}^{k i}$ at which $x_{i}$ is largest. Thus exit costs under the BRM protocol are

$$
C^{B R M}\left(\left\{e_{i}\right\}, \mathcal{B}^{j} \cup \mathcal{B}^{k}\right)=\min \left\{x_{j}^{i j}, x_{k}^{k i}\right\}=\min \left\{\frac{A^{i-j} e_{i}}{A_{i-j}^{i-j}}, \frac{A^{i-k} e_{i}}{A_{i-k}^{i-k}}\right\},
$$

where the last expressions follow from Lemma 3.4. Thus the candidate paths are the same as in the logit model. But since the cost of a given path differs in the two models, the identity of the optimal exit path may differ as well.

Turning to the transition problem, results of Kandori and Rob (1998) imply that under the BRM protocol, the optimal path from $e_{i}$ to $\mathcal{B}^{\ell}, \ell \in\{j, k\}$ is the direct boundary path to 
mixed equilibrium $x^{i \ell} .{ }^{15}$ Thus transition costs are given by

$$
C^{B R M}\left(\left\{e_{i}\right\}, \mathcal{B}^{\ell}\right)=x_{\ell}^{i \ell}=\frac{A^{i-\ell} e_{i}}{A_{i-\ell}^{i-\ell}} .
$$

In particular, in the games considered here, optimal BRM transition paths never pass through the interior of the simplex, as they may in the logit model.

\section{Stationary Distribution Asymptotics and Stochastic Stability}

We now combine results from Section 6 with ones from SS to draw conclusions about the global behavior of the stochastic evolutionary process $\mathbf{X}^{N, \eta}$ in the small noise double limit. Since this process is irreducible, its long run behavior is described by its unique stationary distribution, denoted $\mu^{N, \eta}$. State $x \in X$ is stochastically stable in the small noise double limit if

$$
-\lim _{N \rightarrow \infty} \lim _{\eta \rightarrow 0} \frac{\eta}{N} \log \mu^{N, \eta}(x)=0 .
$$

When the population size $N$ is large enough, the mass placed by $\mu^{N, \eta}$ on any neighborhood of such a state does not vanish at an exponential rate as the noise level $\eta$ approaches zero; see SS Section 6 for further discussion.

As a first application, we characterize the asymptotic behavior of the stationary distributions $\mu^{N, \eta}$ in the small noise double limit when $A$ is a simple three-strategy coordination game and a potential game.

Proposition 7.1. Let $A=C+1 r^{\prime}$ be a simple three-strategy coordination game and a potential game. Let $f(x)=\frac{1}{2} x^{\prime} C x$ be a potential function for $F(x)=A x$, and let $\Delta^{+} f(x)=\max _{i \in S} f\left(e_{i}\right)-f(x)$. Then

$$
\lim _{N \rightarrow \infty} \lim _{\eta \rightarrow 0} \max _{x \in X^{N}}\left|-\frac{\eta}{N} \log \mu^{N, \eta}(x)-\Delta^{+} f(x)\right|=0 .
$$

In words, the proposition says that when $N$ is large, the exponential rate of decay of $\mu^{N, \eta}(x)$ as $\eta$ approaches zero is approximately $N \Delta^{+} f(x)$, where $\Delta^{+} f(x) \geq 0$ is the deficit in

\footnotetext{
${ }^{15}$ For a proof, observe first that in simple three-strategy coordination games, $x_{i}^{i \ell} \geq x_{i}^{*}$ (see Figure 2). The previous paragraph showed that the direct boundary path from $e_{i}$ to $\mathcal{B}^{\ell}$ is optimal among those that do not enter $\mathcal{B}^{h}, h \notin\{i, \ell\}$. This path's cost is $x_{\ell}^{i \ell}=1-x_{i}^{i \ell} \leq 1-x_{i}^{*}=x_{\ell}^{*}+x_{h}^{*}$. But any transition path that enters $\mathcal{B}^{h}$ must have at least this cost, since reaching $\mathcal{B}^{h}$ entails a cost of at least $x_{h}^{i h} \geq x_{h}^{*}$ due to switches from $i$ to $h$, plus a cost of at least $x_{\ell}^{*}$ due to switches from either $i$ or $h$ to $\ell$.
} 
potential of state $x$ relative to the maximizers of potential. Thus the latter states are the stochastically stable states in the small noise double limit.

The proof of Proposition 7.1 combines the analysis in Section 6.1 with the characterization of stationary distribution asymptotics from SS. We abuse notation in what follows by identifying singleton sets with their lone elements (e.g., by writing $C\left(e_{j}, e_{i}\right)$ in place of $\left.C\left(\left\{e_{j}\right\},\left\{e_{i}\right\}\right)\right)$.

Proof. We start by finding the minimal cost $R\left(e_{i}\right)$ of an $e_{i}$-tree. Since the three $e_{i}$-trees are $\left\{\left(e_{j}, e_{i}\right),\left(e_{k}, e_{i}\right)\right\},\left\{\left(e_{k}, e_{j}\right),\left(e_{j}, e_{i}\right)\right\}$, and $\left\{\left(e_{j}, e_{k}\right),\left(e_{k}, e_{i}\right)\right\}$, Corollary 6.3 implies that

$$
\begin{aligned}
R\left(e_{i}\right)= & \min \left\{C\left(e_{j}, e_{i}\right)+C\left(e_{k}, e_{i}\right), C\left(e_{k}, e_{j}\right)+C\left(e_{j}, e_{i}\right), C\left(e_{j}, e_{k}\right)+C\left(e_{k}, e_{i}\right)\right\} \\
= & \min \left\{\left(f\left(e_{j}\right)-f\left(x^{i j}\right)\right)+\left(f\left(e_{k}\right)-f\left(x^{k i}\right)\right),\left(f\left(e_{k}\right)-f\left(x^{j k}\right)\right)+\left(f\left(e_{j}\right)-f\left(x^{i j}\right)\right),\right. \\
& \left.\quad\left(f\left(e_{j}\right)-f\left(x^{j k}\right)\right)+\left(f\left(e_{k}\right)-f\left(x^{k i}\right)\right)\right\} \\
= & f\left(e_{j}\right)+f\left(e_{k}\right)-\max \left\{f\left(x^{i j}\right)+f\left(x^{k i}\right), f\left(x^{j k}\right)+f\left(x^{i j}\right), f\left(x^{j k}\right)+f\left(x^{k i}\right)\right\} \\
= & -f\left(e_{i}\right)+\left(f\left(e_{i}\right)+f\left(e_{j}\right)+f\left(e_{k}\right)-\max \left\{f\left(x^{i j}\right)+f\left(x^{k i}\right), f\left(x^{j k}\right)+f\left(x^{i j}\right), f\left(x^{j k}\right)+f\left(x^{k i}\right)\right\}\right) .
\end{aligned}
$$

In the final expression, the term in parentheses, henceforth denoted $K$, does not depend on the choice of $e_{i}$.

Next, it follows from Lemma 6.2 that for any $x \in X$,

$$
-f\left(e_{i}\right)+C\left(e_{i}, x\right) \geq-f\left(e_{i}\right)+\left(f\left(e_{i}\right)-f(x)\right)=-f(x) .
$$

If $x \in \mathcal{B}^{i}$, then along the straight-line path from $e_{i}$ to $x$ only the optimal strategy $i$ loses mass, so Lemma 6.2 implies that the inequality in (55) binds.

Combining these facts yields

$$
r(x) \equiv \min _{i \in S}\left(R\left(e_{i}\right)+C\left(e_{i}, x\right)\right)=-f(x)+K
$$

Since $A$ is a coordination game, the potential function $f$ is maximized at a pure state, so

$$
\Delta r(x) \equiv r(x)-\min _{y \in X} r(y)=-f(x)-\min _{y \in X}(-f(y))=-f(x)+\max _{i \in S} f\left(e_{i}\right)=\Delta^{+} f(x) .
$$

The proposition thus follows from SS Theorem 6.3.

The close connection between stationary distributions and potential functions in potential games has been understood since the work of Blume (1993,1997). Building on Blume's work, Sandholm (2010c, Corollary 12.2.5) derives statement (54) for a particular specification of the process $\mathbf{X}^{N, \eta}$. In this specification, not only the limit game $F$, but also 
all of the finite-population games $F^{N}$ are assumed to be potential games. This definition ensures that $\mathbf{X}^{N, \eta}$ is reversible for each $(N, \eta)$ pair, and so that each stationary distribution $\mu^{N, \eta}$ admits a simple closed form. ${ }^{16}$ Equation (54) is obtained by taking the limit of these explicit formulas.

In the present analysis, we only assume that the the finite-population games $F^{N}$ converge to a limiting potential game $F$. This assumption does not require $\mathbf{X}^{N, \eta}$ to be reversible, and so explicit expressions for $\mu^{N, \eta}$ are generally unavailable. We describe the asymptotics of the stationary distribution under this weaker assumption by way of the large deviations properties of the stochastic processes. Doing so provides a intuition about the forces behind the selection of the potential maximizer. Since transition costs are determined by differences in potential, the transitions used in every minimum cost tree pass through the same mixed equilibria, so that differences in the trees' costs are due to differences in potential at the trees' roots.

While Proposition 7.1 focuses on simple three-strategy coordination games, similar conclusions can be reached in potential games outside of this class. Benaïm et al. (2014) explore this idea in in the context of large population limits.

The next example provides explicit computations of stochastically stable states under the logit protocol, and compares these predictions with those under the BRM protocol.

Example 7.2. Consider the game $F(x)=A x$ with

$$
A=\left(\begin{array}{ccc}
7 & 0 & 0 \\
2-q & 6 & 0 \\
2 & 0 & 5
\end{array}\right)
$$

where $q \in[0,5)$. For each such $q, A$ is a simple coordination game ${ }^{17}$ with interior equilibrium $x^{*}=\left(\frac{6}{17+q}, \frac{5+q}{17+q}, \frac{6}{17+q}\right)$. The mixed equilibria on the boundary of $X$ are $x^{12}=$ $\left(\frac{6}{11+q}, \frac{5+q}{11+q}, 0\right), x^{23}=\left(0, \frac{5}{11}, \frac{6}{11}\right)$, and $x^{31}=\left(\frac{1}{2}, 0, \frac{1}{2}\right)$. The parameter $q$ is the skew of $A$. Thus when $q=0, A$ is a potential game. ${ }^{18}$

To evaluate stochastic stability, we compute the costs of the direct paths from each pure state to the two adjacent mixed equilibria on the boundary of $X$, as well as the costs of the direct paths from the boundary mixed equilibria to the interior equilibrium $x^{*}$. We present these path costs in Figure 8(i).

Next, when $q$ is positive, we determine whether the optimal path for each counter-

\footnotetext{
${ }^{16}$ See Sandholm (2010c, Theorem 11.5.12).

${ }^{17}$ For the marginal bandwagon property (4), note that $A_{3-1}^{3-2}=5-q$.

${ }^{18}$ In this case, $A$ admits the decomposition $A=C+1 r^{\prime}$ with $C=\left(\begin{array}{lll}5 & 0 & 0 \\ 0 & 6 & 0 \\ 0 & 0 & 5\end{array}\right)$ and $r^{\prime}=\left(\begin{array}{lll}2 & 0 & 0\end{array}\right)$.
} 


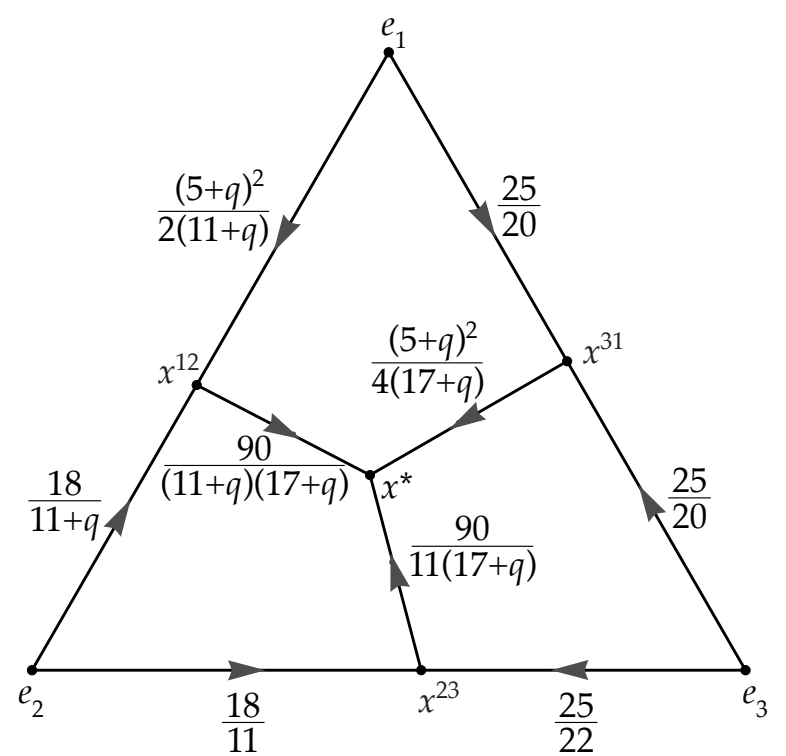

(i) $\operatorname{logit}$

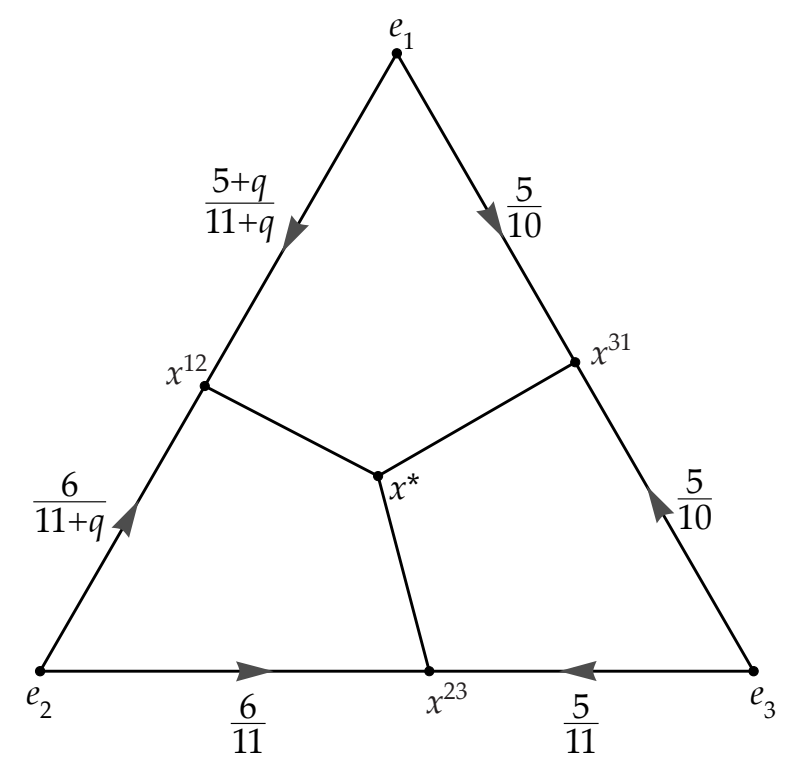

(ii) BRM

Figure 8: The path costs needed to determine transition costs in Example 7.2.

clockwise transition from $e_{j}$ to $\mathcal{B}^{k}$ is the direct path to $x^{j k}$ or the two-segment path via $x^{i j}$ to $x^{*}$ (see Corollary 6.6 and Figures 6 and 7). In the present example, the boundary paths are optimal for every $q \in(0,5)$. Corollary 6.3 implies that they are also optimal when $q=0$.

We then determine the minimum $\operatorname{cost} R\left(e_{i}\right)$ of an $e_{i}$ tree for $i \in\{1,2,3\}$. Simple calculations show that

$$
\begin{aligned}
& R\left(e_{1}\right)=C\left(e_{3}, e_{2}\right)+C\left(e_{2}, e_{1}\right)=\frac{25}{22}+\frac{18}{11+q}, \\
& R\left(e_{2}\right)= \begin{cases}C\left(e_{3}, e_{2}\right)+C\left(e_{1}, e_{2}\right)=\frac{25}{22}+\frac{(5+q)^{2}}{2(11+q)} & \text { if } q \leq \frac{1}{4}(-15+\sqrt{265}) \approx .3197, \\
C\left(e_{3}, e_{2}\right)+C\left(e_{1}, e_{3}\right)=\frac{25}{22}+\frac{25}{20} & \text { otherwise, }\end{cases} \\
& R\left(e_{3}\right)= \begin{cases}C\left(e_{1}, e_{2}\right)+C\left(e_{2}, e_{3}\right)=\frac{(5+q)^{2}}{2(11+q)}+\frac{18}{11} & \text { if } q \leq \frac{5}{22}, \\
C\left(e_{2}, e_{1}\right)+C\left(e_{1}, e_{3}\right)=\frac{18}{11+q}+\frac{25}{20} & \text { otherwise. }\end{cases}
\end{aligned}
$$

Further calculations show that $R\left(e_{2}\right)$ is smallest when $q \in\left[0, \frac{17}{5}\right]$, and that $R\left(e_{1}\right)$ is smallest when $q \in\left[\frac{17}{5}, 5\right)$. Therefore, SS Theorem 6.3 implies that under the logit protocol, state $e_{2}$ is stochastically stable in the small noise double limit in the former case, and state $e_{1}$ is in the latter; both are stochastically stable when $q=\frac{17}{5}$.

We now compare these selection results to those obtained under the BRM protocol. ${ }^{19}$

\footnotetext{
${ }^{19}$ We consider the version of the BRM protocol under which all optimal strategies are chosen with nonnegligible probability. Since the convergence results in SS do not apply to the BRM model, we cannot appeal to them here. But in the present example, the intermediate results needed to establish stochastic
} 
Remark 6.7 states that under this protocol, optimal transition paths in simple coordination games are direct. The BRM costs of the six relevant paths can be read directly from the coordinates of the boundary equilibria; they are shown in Figure 8(ii). Calculations show that the minimal tree costs are

$$
\begin{aligned}
& R^{B R M}\left(e_{1}\right)=C^{B R M}\left(e_{3}, e_{2}\right)+C^{B R M}\left(e_{2}, e_{1}\right)=\frac{5}{11}+\frac{6}{11+q}, \\
& R^{B R M}\left(e_{2}\right)= \begin{cases}C^{B R M}\left(e_{3}, e_{2}\right)+C^{B R M}\left(e_{1}, e_{2}\right)=\frac{5}{11}+\frac{5+q}{11+q} & \text { if } q \leq 1, \\
C^{B R M}\left(e_{3}, e_{2}\right)+C^{B R M}\left(e_{1}, e_{3}\right)=\frac{5}{11}+\frac{5}{10} & \text { otherwise, }\end{cases} \\
& R^{B R M}\left(e_{3}\right)= \begin{cases}C^{B R M}\left(e_{1}, e_{2}\right)+C^{B R M}\left(e_{2}, e_{3}\right)=\frac{5+q}{11+q}+\frac{6}{11} & \text { if } q \leq \frac{11}{23}, \\
C^{B R M}\left(e_{2}, e_{1}\right)+C^{B R M}\left(e_{1}, e_{3}\right)=\frac{6}{11+q}+\frac{5}{10} & \text { otherwise. }\end{cases}
\end{aligned}
$$

Finding the smallest of these costs, we conclude that under the BRM protocol, state $e_{2}$ is stochastically stable when $q \in[0,1)$, and state $e_{1}$ is stochastically stable when $q \in(1,5)$.

To compare predictions under the two protocols, it is useful to focus on the minimal cost trees themselves. Under the logit protocol, three trees have minimal cost for some $q \in[0,5)$ : the $e_{2}$-tree $\left\{\left(e_{3}, e_{2}\right),\left(e_{1}, e_{2}\right)\right\}$ for $q \in[0, \hat{q}], \hat{q} \approx .3197$; the $e_{2}$-tree $\left\{\left(e_{3}, e_{2}\right),\left(e_{1}, e_{3}\right)\right\}$ for $q \in\left[\hat{q}, \frac{17}{5}\right)$; and the $e_{1}$-tree $\left\{\left(e_{3}, e_{2}\right),\left(e_{2}, e_{1}\right)\right\}$ for $q \in\left[\frac{17}{5}, 5\right)$. Under the BRM protocol, only the first and last of these have minimal costs, according to whether $q \in[0,1]$ or $q \in[1,5)$. By way of explanation, notice that as $q$ increases, so does the payoff disadvantage $7-(2-q)=5+q$ of strategy 2 at state $e_{1}$. This causes the cost of the $\left(e_{1}, e_{2}\right)$ transition to grow more rapidly under logit than under BRM, so that the optimal logit $e_{2}$-tree abandons this transition earlier than the optimal BRM $e_{2}$-tree.

Under both protocols, the stochastically stable state switches from equilibrium $e_{2}$ to efficient equilibrium $e_{1}$ as $q$ increases. But the switch occurs sooner for BRM: for $q \in\left(1, \frac{17}{5}\right)$, BRM selects $e_{1}$, while logit selects $e_{2}$. Under BRM, the selection switches once strategy 1 begins to pairwise risk dominate strategy 2 . This would follow from classic results in the absence of strategy 3 , and the fact that transition $\left(e_{3}, e_{2}\right)$, which heads away from $e_{3}$, appears in all BRM minimal cost trees ensures that strategy 3 's presence does not affect the selection. In contrast, as noted above, transition $\left(e_{1}, e_{3}\right)$, which heads into $e_{3}$, is in the logit minimal cost tree for intermediate values of $q$. Its appearance there reflects the advantage of the indirect route from $e_{1}$ to $e_{2}$ via $e_{3}$ over the direct route, and explains why strategy 2 persists as stochastically stable despite being pairwise risk dominated by strategy 1 .

As $q$ increases through $\frac{17}{5}$, the logit minimal cost tree replaces transition $\left(e_{1}, e_{3}\right)$ with

stability follow from elementary considerations, provided that the minimal cost tree is unique. Compare Kandori and Rob $(1995,1998)$. 
transition $\left(e_{2}, e_{1}\right)$, changing the stochastically stable state from $e_{2}$ to $e_{1}$. The former transition must overcome an initial payoff disadvantage of $7-2=5$, compared to $6-0=6$ for the latter, leading the former to be less costly at low values of $q$. As $q$ increases, mixed equilibrium $x^{12}$ moves closer to state $e_{2}$, causing the payoff advantage of strategy 2 over strategy 1 to dissipate more quickly as the state moves from $e_{2}$ toward $e_{1}$. This reduces the cost of the $\left(e_{2}, e_{1}\right)$ transition under the logit protocol, and leads to the replacement of $e_{2}$ by $e_{1}$ as the stochastically stable state.

\section{Discussion}

By combining results from Sandholm and Staudigl (2014) with analyses of optimal control problems, this paper characterized the long-run behavior of a class of stochastic evolutionary processes in the small noise double limit. Our focus here was on evolution in simple three-strategy coordination games under the logit protocol. We conclude by discussing the prospects for extending our analysis to other games and choice rules.

To evaluate these prospects, recall that the running cost appearing in the path cost integral (11) is $L(x, u)=[u]_{+}^{\prime} \Upsilon(F(x))$, where $\Upsilon$ is the unlikelihood function (10) of the revision protocol. The piecewise linearity of $L$ in the control $u=\dot{\phi}_{t}$ ensures that at each state $x$, the optimal choices of $u$ in the HJB equation (17) include extreme points of the control set $Z=\operatorname{conv}\left(\left\{e_{i}-e_{j}: i, j \in S\right\}\right)$. Thus for any game and revision protocol, we expect optimal feedback controls for the exit and transition problems (14) and (15) to partition the state space into regions in which the various basic directions $e_{i}-e_{j}$ are followed.

The logit protocol (1) is particularly convenient because its unlikelihood function (12) is piecewise linear in the payoff vector, and thus piecewise linear in the state when the limit payoff function $F(x)=A x$ is linear. This leads the value functions for problems (14) and (15) to be piecewise quadratic; in particular, they are homogeneous of degree 2 in the displacement of the state from an interior equilibrium $x^{*}$. This ensures that the optimal feedback controls partition the state space into convex sets with common extreme point $x^{*}$, as shown in Figures 4-7. This structure should be preserved by certain other revision protocols. Under the probit protocol (Myatt and Wallace (2003), Dokumaci and Sandholm (2011)), the unlikelihood function is piecewise quadratic. This should lead to value functions that are piecewise cubic-specifically, homogeneous of degree 3 in the displacement of the state from $x^{*}$-so that in the class of games studied here, the boundaries between control regions are again rays emanating from $x^{*}$.

Returning to the logit protocol, the piecewise linearity of running costs $L(x, u)$ in both the control $u$ and the state $x$ suggests that the exit and transition problems can be solved 
beyond the class of simple three-strategy coordination games studied here. The main new consideration in solving control problems (14) and (15) for general linear games is that the state constraints, which require controlled trajectories to stay in the state space $X$, may bind. The fact that these constraints are slack in the games studied here allowed us to appeal to a verification theorem, Theorem 3.1, that does not include such constraints. To handle more general cases, one would need to extend the verification theorem to allow for linear state constraints. For the class of problems generated by the logit protocol, we see no conceptual difficulty in obtaining this extension. Still, the proof of Theorem 3.1 is not simple, and extending it to incorporate state constraints is a challenge we leave for future research.

\section{A. Appendix}

\section{A.1 Proof of Lemma 3.2}

We start by deriving equation (22). Since $V$ is constructed from a feedback control that equals $e_{k}-e_{i}$ in a neighborhood of $x \in \operatorname{int}\left(\mathcal{B}^{i}\right)$, Lemma 3.3 implies that

$$
V\left(x+t\left(e_{i}-e_{k}\right)\right)-V(x)=\gamma\left(x+t\left(e_{i}-e_{k}\right), x\right)=t A^{i-k} x+\frac{t^{2}}{2} A_{i-k}^{i-k}
$$

for $t$ close to zero. Thus

$$
D V(x)\left(e_{k}-e_{i}\right)=-\left.\left(\frac{\mathrm{d}}{\mathrm{d} t} \gamma\left(x+t\left(e_{i}-e_{k}\right), x\right)\right)\right|_{t=0}=A^{k-i} x,
$$

which is equivalent to equation (22).

To verify the HJB equation (19), we must show that the the function to be minimized,

$$
H\left(e_{a}, e_{b}\right)=\left(e_{i}-e_{a}\right)^{\prime} A x+D V(x)^{\prime}\left(e_{a}-e_{b}\right),
$$

is nonnegative at each of the five choices of $\left(e_{a}, e_{b}\right)$ other than $\left(e_{k}, e_{i}\right)$. And indeed,

$$
\begin{aligned}
& H\left(e_{i}, e_{h}\right)=D V(x)\left(e_{i}-e_{h}\right) \geq 0 \text { for } h \in\{j, k\} \text { by }(20), \\
& H\left(e_{j}, e_{i}\right)=\left(D V(x)-(A x)^{\prime}\right)\left(e_{j}-e_{i}\right)=\left(D V(x)-(A x)^{\prime}\right)\left(e_{j}-e_{k}\right) \geq 0 \text { by (22) and (21), } \\
& H\left(e_{k}, e_{j}\right)=\left(e_{i}-e_{k}\right)^{\prime} A x+D V(x)\left(e_{k}-e_{j}\right)=D V(x)\left(e_{i}-e_{j}\right) \geq 0 \text { by (22) and (20), and } \\
& H\left(e_{j}, e_{k}\right)=\left(e_{i}-e_{j}\right)^{\prime} A x+D V(x)\left(e_{j}-e_{k}\right) \geq\left(e_{i}-e_{k}\right)^{\prime} A x \geq 0 \text { by (21) and the fact that } x \in \mathcal{B}^{i} .
\end{aligned}
$$




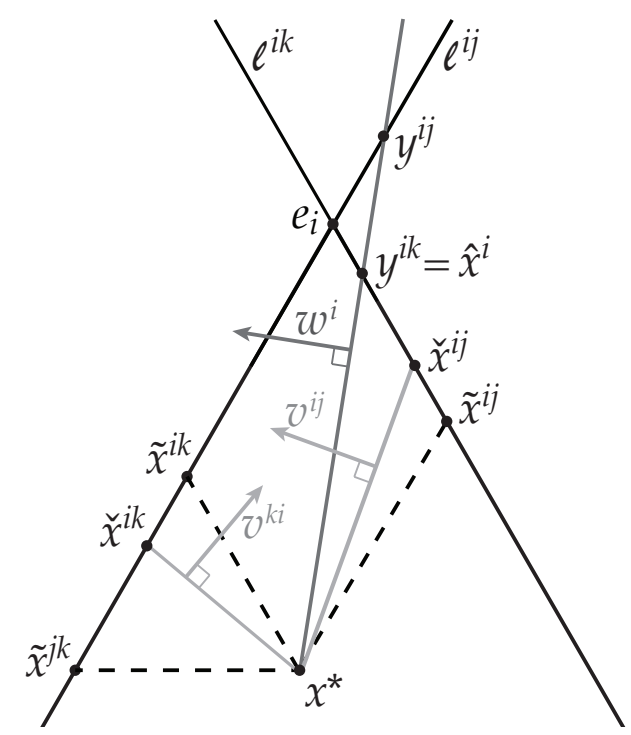

Figure 9: The construction of $\hat{x}^{i}$ when it is on face $e_{k} e_{i}$ and $Q>0$.

\section{A.2 Proof of Lemma 5.1}

For concreteness, we assume that $Q \geq 0$. The proof when $Q<0$ is essentially the same, but with strategies $j$ and $k$ interchanged.

Let $\tilde{x}^{i k}=x^{*}+x_{k}^{*}\left(e_{i}-e_{k}\right)=\left(x_{i}^{*}+x_{k}^{*}\right) e_{i}+x_{j}^{*} e_{j}$ and $\tilde{x}^{i j}=x^{*}+x_{j}^{*}\left(e_{i}-e_{j}\right)=\left(x_{i}^{*}+x_{j}^{*}\right) e_{i}+x_{k}^{*} e_{k}$ (see Figure 9). We start by establishing

Lemma A.1. $V^{k}\left(\tilde{x}^{i k}\right)>V^{j}\left(\tilde{x}^{i k}\right)$ and $V^{k}\left(\tilde{x}^{i j}\right)<V^{j}\left(\tilde{x}^{i j}\right)$.

Proof. Observe that

$$
\begin{aligned}
V^{k}\left(\tilde{x}^{i k}\right)-V^{j}\left(\tilde{x}^{i k}\right) & =\frac{1}{2}\left(\frac{\left(A^{i-k}\left(x^{*}+x_{k}^{*}\left(e_{i}-e_{k}\right)\right)\right)^{2}}{A_{i-k}^{i-k}}-\frac{\left(A^{i-j}\left(x^{*}+x_{k}^{*}\left(e_{i}-e_{k}\right)\right)\right)^{2}}{A_{i-j}^{i-j}}\right) \\
& =\frac{\left(x_{k}^{*}\right)^{2}}{2}\left(A_{i-k}^{i-k}-\frac{\left(A_{i-k}^{i-j}\right)^{2}}{A_{i-j}^{i-j}}\right) .
\end{aligned}
$$

Thus to prove the first inequality, it suffices to show that $A_{i-j}^{i-j} A_{i-k}^{i-k}-\left(A_{i-k}^{i-j}\right)^{2}>0$. And indeed,

$$
A_{i-j}^{i-j} A_{i-k}^{i-k}-A_{i-k}^{i-j} A_{i-k}^{i-j}=A_{i-j}^{i-j} A_{i-k}^{i-k}-A_{i-j}^{i-j} A_{i-k}^{i-j}-A_{j-k}^{i-j} A_{i-k}^{i-j}=A_{j-k}^{j-i} A_{i-k}^{i-j}+A_{i-j}^{i-j} A_{k-i}^{k-j}>0 .
$$

Interchanging $j$ and $k$ in these calculations proves the second inequality.

Next, let $\ell^{i j}=\left\{s e_{i}+(1-s) e_{j}: s \in \mathbb{R}\right\}$. The directional derivative of the quadratic function $V^{k}-V^{j}$ along this line is evaluated as follows: 


$$
\begin{aligned}
\left(D V^{k}(x)-D V^{j}(x)\right)\left(e_{i}-e_{j}\right) & =\left(\frac{A^{i-k} x}{A_{i-k}^{i-k}} A^{i-k}-\frac{A^{i-j} x}{A_{i-j}^{i-j}} A^{i-j}\right)\left(e_{i}-e_{j}\right) \\
& =\frac{A^{i-k} x}{A_{i-k}^{i-k}} A_{i-j}^{i-k}-A^{i-j} x \\
& =\frac{1}{A_{i-k}^{i-k}}\left(A_{i-j}^{i-k} A^{i-k}-A_{i-k}^{i-k} A^{i-j}\right) x \\
& =\frac{1}{A_{i-k}^{i-k}}\left(-A_{k-j}^{k-i} A^{i}+A_{i-k}^{i-k} A^{j}-A_{i-j}^{i-k} A^{k}\right) x \\
& =-\left(v^{k i}\right)^{\prime} x .
\end{aligned}
$$

Thus on $\ell^{i j}, V^{k}-V^{j}$ is concave and is maximized at the unique state $\breve{x}^{i k}$ satisfying $\left(v^{k i}\right)^{\prime} x=0$ (see Figure 9).

Recall that $\tilde{x}^{i k}=x^{*}+x_{k}^{*}\left(e_{i}-e_{k}\right)=x_{j}^{*} e_{j}+\left(x_{i}^{*}+x_{k}^{*}\right) e_{i} \in \ell^{i j}$, and let $\tilde{x}^{j k}=x^{*}+x_{k}^{*}\left(e_{j}-e_{k}\right)=$ $x_{i}^{*} e_{i}+\left(x_{j}^{*}+x_{k}^{*}\right) e_{j} \in \ell^{i j}$. Since $Q \geq 0$, equations (35) and (36) and inequality (37) imply that $\left(v^{k i}\right)^{\prime} \tilde{x}^{i k}=x_{k}^{*} Q \geq 0$ and that $\left(v^{k i}\right)^{\prime} \tilde{x}^{j k}<0$. Thus $\check{x}^{i k}$ lies between $\tilde{x}^{i k}$ and $\tilde{x}^{j k}$, and is equal to the former if and only if $Q=0$ (again, see Figure 9). Since $V^{k}\left(\tilde{x}^{i k}\right)>V^{j}\left(\tilde{x}^{i k}\right)$ by Lemma A.1 and since $V^{k}-V^{j}$ is concave quadratic on $\ell^{i j}$, we have

Lemma A.2. There is a unique state $y^{i j} \in \ell^{i j}$ with $y_{i}^{i j}>\check{x}_{i}^{i k} \geq x_{i}^{*}$ such that $V^{k}\left(y^{i j}\right)=V^{j}\left(y^{i j}\right)$.

Next, we consider directional derivative of the quadratic function $V^{k}-V^{j}$ along line $\ell^{i k}=\left\{s e_{i}+(1-s) e_{k}: s \in \mathbb{R}\right\}$. A calculation similar to (56) shows that

$$
\left(D V^{k}(x)-D V^{j}(x)\right)\left(e_{i}-e_{k}\right)=\frac{1}{A_{i-j}^{i-j}}\left(A_{i-j}^{i-j} A^{i-k}-A_{i-k}^{i-j} A^{i-j}\right) x=\left(v^{i j}\right)^{\prime} x
$$

Thus on $\ell^{i k}, V^{k}-V^{j}$ is convex and is minimized at the unique state $\check{x}^{i j}$ on $\ell^{i k}$ satisfying $\left(v^{i j}\right)^{\prime} x=0$ (once again, see Figure 9). Since $Q \geq 0$, equations (35) and (36) and inequality (37) imply that $\check{x}^{i j}=\tilde{x}^{i j}+c\left(e_{i}-e_{k}\right)$ for some $c \geq 0$, with equality only if and only if $Q=0$. Since $V^{k}\left(\tilde{x}^{i j}\right)<V^{j}\left(\tilde{x}^{i j}\right)$ by Lemma A.1 and since $V^{k}-V^{j}$ is convex quadratic on $\ell^{i k}$, we have Lemma A.3. There is a unique state $y^{i k} \in \ell^{i k}$ with $y_{i}^{i k}>\check{x}_{i}^{i j} \geq \tilde{x}_{i}^{i j}$ such that $V^{k}\left(y^{i k}\right)=V^{j}\left(y^{i k}\right)$.

To complete the proof, we use the homogeneity of degree 2 of $V^{j}(x)$ and $V^{k}(x)$ in the displacement $z=x-x^{*}$ of $x$ from $x^{*}$. Specifically, for $z \in T X$ and $s \in \mathbb{R}$, we have

$$
V^{k}\left(x^{*}+s z\right)-V^{j}\left(x^{*}+s z\right)=V^{k}(s z)-V^{j}(s z)=s^{2}\left(V^{k}(z)-V^{j}(z)\right)=s^{2}\left(V^{k}\left(x^{*}+z\right)-V^{j}\left(x^{*}+z\right)\right) .
$$

Thus if $V^{k}\left(x^{*}+z\right)=V^{j}\left(x^{*}+z\right)$, then $V^{k}\left(x^{*}+s z\right)=V^{j}\left(x^{*}+s z\right)$ for all $s \in \mathbb{R}$. It therefore follows from Lemmas A.2 and A.3 that $y^{i j}$ and $y^{i k}$ are collinear with $x^{*}$ (see Figure 9), and 
so that both of these points satisfy (39), (40), and (41). It could be that $y^{i j}=y^{i k}=e_{i}$, in which case we choose $\hat{x}^{i}=e_{i}$. Otherwise, exactly one of $y^{i j}$ and $y^{i k}$ is in $X$, in which case we choose $\hat{x}^{i}$ to be this state. This completes the proof of the lemma.

\section{A.3 Proof of Proposition 6.5}

\section{A.3.1 Preliminary calculations}

We start by stating a counterpart of Lemma 3.4 for paths along $\mathcal{B}^{i j}$ to $x^{*}$.

Lemma A.4. If $y \in \mathcal{B}^{i j}$, then

$$
\begin{aligned}
& y=x^{*}+d^{\zeta}(y) \zeta^{i j}, \text { where } d^{\zeta}(y)=x_{k}^{*}-y_{k}, \text { and } \\
& \gamma\left(y, x^{*}\right)=\frac{1}{2} d^{\zeta}(y)^{2} A_{\zeta^{\zeta}}^{\zeta} \text { where } A_{\zeta}^{\zeta}=\left(\zeta^{i j}\right)^{\prime} A \zeta^{i j}>0 .
\end{aligned}
$$

Proof. Since $y \in \operatorname{conv}\left(\left\{x^{i j}, x^{*}\right\}\right),(27)$ implies that we can write $y=x^{*}+d \zeta^{i j}$ for some $d>0$. Then (28) implies that $y_{k}=x_{k}^{*}-d$, which yields (57), and Lemma 3.3 implies that

$$
\begin{aligned}
\gamma\left(y, x^{*}\right) & =\left(y-x^{*}\right)^{\prime} A\left(\frac{y+x^{*}}{2}\right) \\
& =d^{\zeta}(y)\left(\zeta^{i j}\right)^{\prime} A\left(x^{*}+\frac{1}{2} d^{\zeta}(y) \zeta^{i j}\right) \\
& =\frac{1}{2} d^{\zeta}(y)^{2} A_{\zeta}^{\zeta} .
\end{aligned}
$$

Lemma A.4 gives an expression for $d^{\zeta}(y)$ that is affine in $y$. To match Lemma 3.4, one can instead write $d^{\zeta}(y)=\left(\zeta^{i j}\right)^{\prime} A y / A_{\zeta}^{\zeta}$. The key point is that either way, $d^{\zeta}\left(x^{*}+z\right)$ is linear in the displacement $z$.

Next we give explicit expressions for each piece of the value function and their derivatives.

$$
\begin{aligned}
V^{1}(x) & =\gamma\left(x, \omega^{i k}(x)\right)=\frac{1}{2} d^{i k}(x)^{2} A_{i-k}^{i-k}=\frac{1}{2} \frac{\left(A^{i-k} x\right)^{2}}{A_{i-k}^{i-k}}, \text { so } \\
D V^{1}(x) & =\frac{A^{i-k} x}{A_{i-k}^{i-k}} A^{i-k} ; \\
V^{2}(x) & =\gamma\left(x, \chi^{i k}(x)\right)+\gamma\left(\chi^{i k}(x), x^{*}\right) \\
& =\delta^{i k}(x) A^{i-k} \chi^{i k}(x)+\frac{1}{2} \delta^{i k}(x)^{2} A_{i-k}^{i-k}+\frac{1}{2} d^{\zeta}\left(\chi^{i k}(x)\right)^{2} A_{\zeta^{\prime}}^{\zeta} \text { so }
\end{aligned}
$$




$$
\begin{aligned}
& D V^{2}(x)=\delta^{i k}(x) A^{i-k} D \chi^{i k}(x)+A^{i-k} \chi^{i k}(x) D \delta^{i k}(x)+A_{i-k}^{i-k} \delta^{i k}(x) D \delta^{i k}(x) \\
& +A_{\zeta}^{\zeta} d^{\zeta}\left(\chi^{i k}(x)\right) D d^{\zeta}\left(\chi^{i k}(x)\right) D \chi^{i k}(x) \\
& V^{3}(x)=\gamma\left(x, \chi^{j i}(x)\right)+\gamma\left(\chi^{j i}(x), x^{*}\right)=\frac{1}{2} d^{j i}(x)^{2} A_{j-i}^{j-i}+\frac{1}{2} d^{\zeta}\left(\chi^{j i}(x)\right)^{2} A_{\zeta}^{\zeta} \text {, so } \\
& D V^{3}(x)=A_{j-i}^{j-i} d^{j i}(x) D d^{j i}(x)+A_{\zeta}^{\zeta} d^{\zeta}\left(\chi^{j i}(x)\right) D d^{\zeta}\left(\chi^{j i}(x)\right) D \chi^{j i}(x) \text {; and } \\
& V^{4}(x)=\gamma\left(x, \omega^{j k}(x)\right)=\frac{1}{2} d^{j k}(x)^{2} A_{j-k}^{j-k}=\frac{1}{2} \frac{\left(A^{j-k} x\right)^{2}}{A_{j-k}^{j-k}} \text {, so } \\
& D V^{4}(x)=\frac{A^{j-k} x}{A_{j-k}^{j-k}} A^{j-k} .
\end{aligned}
$$

The functions above are expressed in terms of derivatives of linear functions from Section 6.2 and Lemma A.4. These derivatives are written explicitly as follows:

$$
\begin{aligned}
& D \delta^{i k}(x)=\frac{A^{i-j}}{A_{i-k}^{i-j}}, \\
& D \chi^{i k}(x)=I+\left(e_{k}-e_{i}\right) D \delta^{i k}(x)=I+\left(e_{k}-e_{i}\right) \frac{A^{i-j}}{A_{i-k}^{i-j},} \\
& D d^{j i}(x)=\frac{A^{j-i}}{A_{j-i}^{j-i}}, \\
& D \chi^{j i}(x)=I+\left(e_{i}-e_{j}\right) D d^{j i}(x)=I+\left(e_{i}-e_{j}\right) \frac{A^{j-i}}{A_{j-i}^{j-i}}, \text { and } \\
& D d^{\zeta}(y)=-e_{k}^{\prime} \text { for } y \in \mathcal{B}^{i j} .
\end{aligned}
$$

The remainder of the proof makes extensive use of the expressions above. While the algebraic presentation below may look complicated, many of the arguments are quite simple when interpreted geometrically.

\section{A.3.2 Proof of Lemma 6.4}

We consider the behavior of the quadratic function $V^{4}-V^{3}$ on the line $\ell^{i j}=\left\{s e_{i}+(1-\right.$ $\left.s) e_{j}: s \in \mathbb{R}\right\}$. To begin, note that since $D \chi^{j i}(x)\left(e_{j}-e_{i}\right)=0$, a calculation similar to (56) shows that

$$
\left(D V^{4}(x)-D V^{3}(x)\right)\left(e_{j}-e_{i}\right)=\frac{1}{A_{j-k}^{j-k}}\left(A_{j-i}^{j-k} A^{j-k}-A_{j-k}^{j-k} A^{j-i}\right) x=-\left(v^{j k}\right)^{\prime} x .
$$




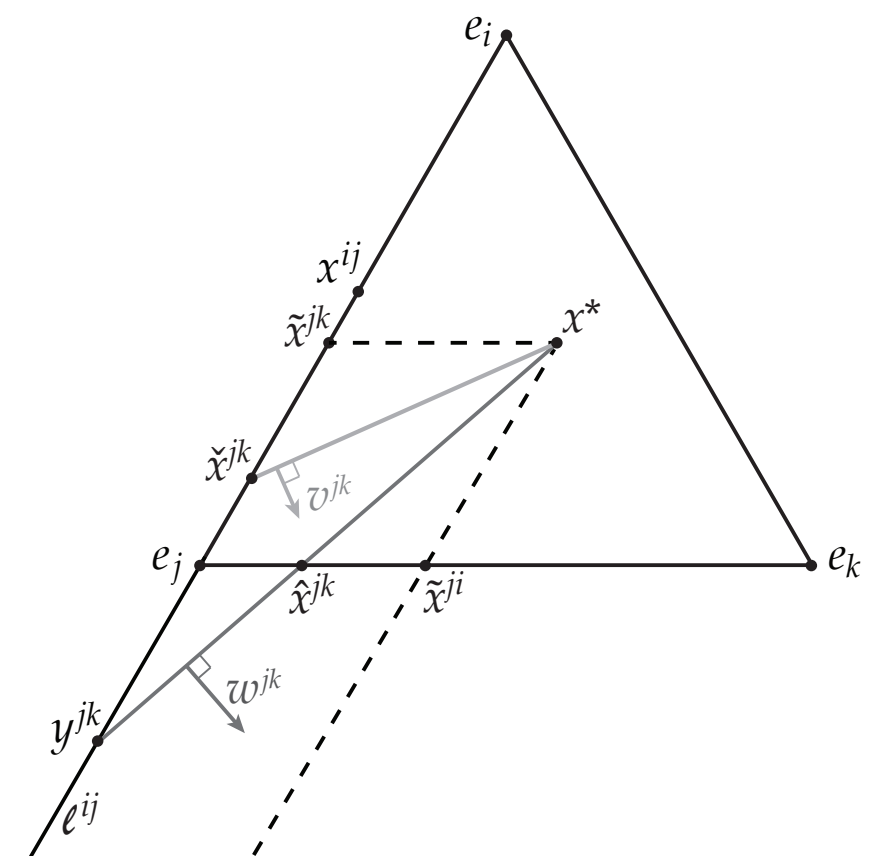

Figure 10: The construction of $\hat{x}^{j k}$ when it is on face $e_{j} e_{k}$.

Since $e_{j}-e_{i}$ is tangent to $\ell^{i j}$, it follows that $V^{4}-V^{3}$ is concave on $\ell^{i j}$ and reaches its maximum on this line at the unique state on $\ell^{i j}$ satisfying $\left(v^{j k}\right)^{\prime} x=0$. We denote this state by $\check{x}^{j k}$ (see Figure 10).

Let $\tilde{x}^{j k}=x^{*}+x_{k}^{*}\left(e_{j}-e_{k}\right) \in \ell^{i j}$. We show that $V^{4}\left(\tilde{x}^{j k}\right)-V^{3}\left(\tilde{x}^{j k}\right)>0$. Observe that $d^{j k}\left(\tilde{x}^{j k}\right)=x_{k}^{*} d^{\zeta}\left(\tilde{x}^{j k}\right)=x_{k}^{*}$, and

$$
d^{j i}\left(\tilde{x}^{j k}\right)=\frac{A^{j-i}\left(x^{*}+x_{k}^{*}\left(e_{j}-e_{k}\right)\right)}{A_{j-i}^{j-i}}=x_{k}^{*} \frac{A_{j-k}^{j-i}}{A_{j-i}^{j-i}},
$$

we have that

$$
V^{4}\left(\tilde{x}^{j k}\right)-V^{3}\left(\tilde{x}^{j k}\right)=\frac{1}{2}\left(x_{k}^{*}\right)^{2} A_{i-k}^{j-k}-\left(\frac{1}{2}\left(x_{k}^{*} \frac{A_{j-k}^{j-i}}{A_{j-i}^{j-i}}\right)^{2} A_{j-i}^{j-i}+\frac{1}{2}\left(x_{k}^{*}\right)^{2} A_{\zeta}^{\zeta}\right) .
$$

Since

$$
A^{j-i} \zeta^{i j}=\frac{1}{x_{k}^{*}} A^{j-i}\left(x^{i j}-x^{*}\right)=\frac{1}{x_{k}^{*}} A^{j-i} x^{i j}=0,
$$

and $\zeta^{i j}+\zeta_{i}^{i j}\left(e_{j}-e_{i}\right)=e_{j}-e_{k}$, and using expression (28) for $\zeta^{i j}$, we have 


$$
\begin{aligned}
A_{\zeta}^{\zeta} & =\left(\zeta^{i j}\right)^{\prime} A \zeta^{i j} \\
& =\left(e_{j}-e_{k}\right)^{\prime} A \zeta^{i j} \\
& =A^{j-k}\left(\frac{A_{j-k}^{j-i}}{A_{j-i}^{j-i}}\left(e_{i}-e_{k}\right)+\frac{A_{i-k}^{i-j}}{A_{j-i}^{j-i}}\left(e_{j}-e_{k}\right)\right) \\
& =\frac{1}{A_{j-i}^{j-i}}\left(A_{i-k}^{j-k} A_{j-k}^{j-i}+A_{j-k}^{j-k} A_{i-k}^{i-j}\right) .
\end{aligned}
$$

Thus continuing from (58), we have

$$
\begin{aligned}
\frac{2 A_{j-i}^{j-i}}{\left(x_{k}^{*}\right)^{2}}\left(V^{4}\left(\tilde{x}^{j k}\right)-V^{3}\left(\tilde{x}^{j k}\right)\right) & =A_{j-k}^{j-k} A_{j-k}^{j-i}-\left(A_{j-k}^{j-i}\right)^{2}-A_{i-k}^{j-k} A_{j-k}^{j-i}-A_{j-k}^{j-k} A_{i-k}^{i-j} \\
& =A_{j-k}^{j-k} A_{j-k}^{j-i}-\left(A_{j-k}^{j-i}\right)^{2}-A_{i-k}^{j-k} A_{j-k}^{j-i} \\
& =A_{j-k}^{j-i}\left(A_{j-i}^{j-k}-A_{j-k}^{j-i}\right) \\
& =A_{j-k}^{j-i} Q \\
& >0 .
\end{aligned}
$$

as claimed.

Since $\left(v^{j k}\right)^{\prime} \check{x}^{j k}=0$ and $\left(v^{i k}\right)^{\prime}\left(e_{i}-e_{j}\right)>0$ (see (38)), it follows that $\check{x}^{j k}=\tilde{x}^{j k}+c\left(e_{j}-e_{i}\right)$ for some $c>0$. Thus as one proceeds along $\ell^{i j}$ in direction $e_{j}-e_{i}$ starting from $\tilde{x}^{j k}$, the function $V^{4}-V^{3}$ starts at a positive value, increases until reaching its maximum at $\check{x}^{j k}$, and then decreases, ultimately approaching $-\infty$. Thus there is a unique point $y^{j k}=\check{x}^{j k}+b\left(e_{j}-e_{i}\right) \in \ell^{i j}$ with $b>0$ at which $V^{4}(x)-V^{3}(x)=0$ (see Figure 10).

If $y_{i}^{j k} \geq 0$, so that $y^{j k}$ is in $X$, then we let $\hat{x}^{j k}=y^{j k}$, and this point clearly satisfies (50), (49), and (51). If instead $y_{i}^{j k}<0$, we let

$$
\hat{x}^{j k}=\frac{x_{i}^{*}}{x_{i}^{*}-y_{i}^{j k}} y_{i}^{j k}+\frac{-y_{i}^{j k}}{x_{i}^{*}-y_{i}^{j k}} x^{*},
$$

which is the point on the segment between $y^{j k}$ and $x^{*}$ whose $i$ th component is 0 (see Figure 10). Since equality (51) and inequality (50) hold at $y^{j k}$ and are preserved along rays from $x^{*}$, they continue to hold at $\hat{x}^{j k}$, with a strict inequality in the case of (50). And since $\hat{x}_{k}^{j k}$ is a strictly convex combination of $x_{k}^{*}$ and $-y_{k}^{j k}>0$, we have $\hat{x}_{k}^{j k}<x_{k}^{*}$, which is inequality (49). This completes the proof of the lemma. 


\section{A.3.3 Continuity and piecewise smoothness of $V^{*}$}

Lemma A.5 shows that the value function $V^{*}$ is continuous on the boundary between the third and fourth cases of definition (52).

Lemma A.5. If $x \in \mathcal{B}^{j}$ and $\left(w^{j k}\right)^{\prime} x=0$, then $V^{3}(x)=V^{4}(x)$.

Proof. Since $\left(w^{j k}\right)^{\prime} x=0$ and $w^{j k}=x^{*} \times\left(\hat{x}^{j k}-x^{*}\right)$, we can write $x=x^{*}+r\left(\hat{x}^{j k}-x^{*}\right)$ for some $r \in[0,1]$. By condition (51) and the expressions for $V^{3}$ and $V^{4}$ above, it is enough to show that $d^{j k}(x)=r d^{j k}\left(\hat{x}^{j k}\right), d^{j i}(x)=r d^{j i}\left(\hat{x}^{j k}\right)$, and $d^{\zeta}\left(\chi^{j i}(x)\right)=r d^{\zeta}\left(\chi^{j i}(x)\right)$. And indeed, the fact that $A x^{*}$ is a multiple of $\mathbf{1}$ implies that

$$
\begin{aligned}
& d^{j k}(x)=\frac{A^{j-k} x}{A_{j-k}^{j-k}}=r \frac{A^{j-k} \hat{x}^{j k}}{A_{j-k}^{j-k}}=r d^{j k}\left(\hat{x}^{j k}\right) \text { and } \\
& d^{j i}(x)=\frac{A^{j-i} x}{A_{j-i}^{j-i}}=r \frac{A^{j-i} \hat{x}^{j k}}{A_{j-i}^{j-i}}=r d^{j i}\left(\hat{x}^{j k}\right),
\end{aligned}
$$

while the third equality follows from the fact that

$$
d^{\zeta}\left(\chi^{j i}(x)\right)=x_{k}^{*}-e_{k}^{\prime}\left(x+\left(e_{i}-e_{j}\right) d^{j i}(x)\right)=x_{k}^{*}-x_{k} .
$$

Lemmas A.6 and A.7 establish differentiability of $V^{*}$ on the boundaries between the first and second and the second and third cases of definition (52).

Lemma A.6. If $\tilde{x} \in \mathcal{B}^{i}$ satisfies $\tilde{x}=x^{*}+d\left(e_{i}-e_{k}\right)$ for some $d \geq 0$, then $D V^{1}(\tilde{x})=D V^{2}(\tilde{x})=d A^{i-k}$.

Proof. Note first that

$$
D V^{1}(\tilde{x})=\frac{A^{i-k} \tilde{x}}{A_{i-k}^{i-k}} A^{i-k}=\frac{A^{i-k}\left(x^{*}+d\left(e_{i}-e_{k}\right)\right)}{A_{i-k}^{i-k}} A^{i-k}=d A^{i-k} .
$$

To compute $D V^{2}(\tilde{x})$, use the definition of $\chi^{i k}$ and Lemma A.4 (or draw a picture) to show that $\delta^{i k}(\tilde{x})=d, \chi^{i k}(\tilde{x})=x^{*}$, and $d^{\zeta}\left(x^{*}\right)=0$. Then since $A^{i-k} x^{*}=0$, we have that

$$
\begin{aligned}
D V^{2}(\tilde{x}) & =\delta^{i k}(\tilde{x}) A^{i-k} D \chi^{i k}(\tilde{x})+A_{i-k}^{i-k} \delta^{i k}(\tilde{x}) D \delta^{i k}(\tilde{x}) \\
& =d A^{i-k}\left(I+\left(e_{k}-e_{i}\right) \frac{A^{i-j}}{A_{i-k}^{i-j}}\right)+A_{i-k}^{i-k} d \frac{A^{i-j}}{A_{i-k}^{i-j}} \\
& =d A^{i-k}+d A_{k-i}^{i-k} \frac{A^{i-j}}{A_{i-k}^{i-j}}+A_{i-k}^{i-k} d \frac{A^{i-j}}{A_{i-k}^{i-j}} \\
& =d A^{i-k} .
\end{aligned}
$$


Lemma A.7. If $y \in \mathcal{B}^{i j}$, then $D V^{2}(y)=D V^{3}(y)=(A y)^{\prime}$ (as linear forms on $T X$ ).

Proof. Since $y \in \mathcal{B}^{i j}, \delta^{i k}(y)=0, \chi^{i k}(y)=y$, and $d^{\zeta}(y)=d$. Thus

$$
\begin{aligned}
D V^{2}(y) & =A^{i-k} \chi^{i k}(y) D \delta^{i k}(y)+A_{\zeta}^{\zeta} d^{\zeta}\left(\chi^{i k}(y)\right) D d^{\zeta}\left(\chi^{i k}(y)\right) D \chi^{i k}(y) \\
& =A^{i-k} y \frac{A^{i-j}}{A_{i-k}^{i-j}}+A_{\zeta}^{\zeta}\left(x_{k}^{*}-y_{k}\right)\left(-e_{k}^{\prime}\left(I+\left(e_{k}-e_{i}\right) \frac{A^{i-j}}{A_{i-k}^{i-j}}\right)\right) \\
& =A^{i-k} y \frac{A^{i-j}}{A_{i-k}^{i-j}}+A_{\zeta}^{\zeta}\left(x_{k}^{*}-y_{k}\right)\left(-e_{k}^{\prime}-\frac{A^{i-j}}{A_{i-k}^{i-j}}\right) \\
& =\frac{A^{i-k} y-A_{\zeta}^{\zeta}\left(x_{k}^{*}-y_{k}\right)}{A_{i-k}^{i-j}} A^{i-j}-A_{\zeta}^{\zeta}\left(x_{k}^{*}-y_{k}\right) e_{k}^{\prime} .
\end{aligned}
$$

Thus

$$
D V^{2}(y)\left(e_{i}-e_{k}\right)=A^{i-k} y-A_{\zeta}^{\zeta}\left(x_{k}^{*}-y_{k}\right)+A_{\zeta}^{\zeta}\left(x_{k}^{*}-y_{k}\right)=A^{i-k} y=(A y)^{\prime}\left(e_{i}-e_{k}\right),
$$

and since $A^{i-j} \zeta^{i j}=0$ (see (59)) and

$$
\left(\zeta^{i j}\right)^{\prime} A y=\left(\zeta^{i j}\right)^{\prime} A\left(x^{*}+d^{\zeta}(y) \zeta^{i j}\right)=A_{\zeta}^{\zeta}\left(x_{k}^{*}-y_{k}\right)
$$

we have

$$
D V^{2}(y) \zeta^{i j}=A_{\zeta}^{\zeta}\left(x_{k}^{*}-y_{k}\right)=(A y)^{\prime} \zeta^{i j}
$$

Since $e_{i}-e_{k}$ and $\zeta^{i j}$ span $T X$, we conclude that $D V^{2}(y)=(A y)^{\prime}$.

Again using $\delta^{j i}(y)=0$ and $\chi^{j i}(y)=y$, we have

$$
\begin{aligned}
D V^{3}(y) & =A_{\zeta}^{\zeta} d^{\zeta}\left(\chi^{j i}(y)\right) D d^{\zeta}\left(\chi^{j i}(y)\right) D \chi^{j i}(y) \\
& =A_{\zeta}^{\zeta}\left(x_{k}^{*}-y_{k}\right)\left(-e_{i}^{\prime}\left(I+\left(e_{i}-e_{j}\right) \frac{A^{j-i}}{A_{j-i}^{j-i}}\right)\right) \\
& =-A_{\zeta}^{\zeta}\left(x_{k}^{*}-y_{k}\right) e_{k}^{\prime} .
\end{aligned}
$$

Thus

$$
\begin{aligned}
& D V^{3}(y)\left(e_{i}-e_{j}\right)=0=(A y)^{\prime}\left(e_{i}-e_{j}\right), \text { and } \\
& D V^{3}(y) \zeta^{i j}=A_{\zeta}^{\zeta}\left(x_{k}^{*}-y_{k}\right)=(A y)^{\prime} \zeta^{i j} .
\end{aligned}
$$


Thus since $e_{i}-e_{j}$ and $\zeta^{i j}$ span $T X$, we conclude that $D V^{3}(y)=(A y)^{\prime}$.

\section{A.3.4 Checking the HJB equation}

To complete the proof of Proposition 6.5, we need to show that the HJB equation (19) is satisfied at all states at which $V^{*}$ is $C^{1}$. In the first case of the definition (52) of $V^{*}$, this follows from Lemma 4.1 and the fact that $Q>0$, since equation (36) implies that $\left(v^{k i}\right)^{\prime} x \geq 0$ when $x \in \mathcal{B}^{i}$ and $x_{j} \leq x_{j}^{*}$ (see Figure 3(ii)).

Similarly, in the the fourth case of definition (52), the HJB equation follows from Lemma 4.1 (with the roles of $i$ and $j$ reversed) and Lemma 6.4, which ensures that $\left(v^{j k}\right)^{\prime} x \geq 0$ when $x \in \mathcal{B}^{j}$ and $\left(w^{j k}\right)^{\prime} x \geq 0$ (see Figure 10).

To handle the two remaining cases of definition (52), we apply Lemma 3.2. Observe that the regions defined by these cases are convex cones in aff $(X)$ emanating from $x^{*}$. Also, the expressions for $D V^{2}(x)$ and $D V^{3}(x)$ in Appendix A.3.1 imply that within each of these regions, the function to be minimized in the HJB equation (19) is linear in the displacement $z=x-x^{*}$ of $x$ from $x^{*}$. Therefore, to establish inequalities (20) and (21) from Lemma 3.2 for all the states in one of these cones, it is enough to do so at three states: $x^{*}$, and one state from each edge of the cone. Since we have shown that $V^{*}$ is $C^{1}$ on the boundaries between the first and second and the second and third cases of (52), this analysis also establishes that the HJB equation (19) holds on these boundaries.

For the second case of definition (52), we show that inequalities (20) and (21) hold at states $x^{*}, x^{i j}$, and $\tilde{x}^{i k}=x^{*}+x_{k}^{*}\left(e_{i}-e_{k}\right)=\left(x_{i}^{*}+x_{k}^{*}\right) e_{i}+x_{j}^{*} e_{j}$ :

$$
\begin{aligned}
& D V^{2}\left(x^{*}\right)=D V^{2}\left(x^{*}\right)-\left(A x^{*}\right)^{\prime}=\mathbf{0}^{\prime} \text { (as a linear form on TX); } \\
& D V^{2}\left(x^{i j}\right)\left(e_{i}-e_{k}\right)=A^{i-k} x^{i j}>0, \\
& D V^{2}\left(x^{i j}\right)\left(e_{i}-e_{j}\right)=A^{i-j} x^{i j}=0, \\
& \left.D V^{2}\left(x^{i j}\right)-\left(A x^{i j}\right)^{\prime}=\mathbf{0}^{\prime} \text { (as a linear form on } T X\right) ; \\
& D V^{2}\left(\tilde{x}^{i k}\right)\left(e_{i}-e_{k}\right)=x_{k}^{*} A_{i-k}^{i-k}>0, \\
& D V^{2}\left(\tilde{x}^{i k}\right)\left(e_{i}-e_{j}\right)=x_{k}^{*} A_{i-j}^{i-k}>0, \\
& \left(D V^{2}\left(\tilde{x}^{i k}\right)-\left(A \tilde{x}^{i k}\right)^{\prime}\right)\left(e_{k}-e_{j}\right)=\left(D V^{1}\left(\tilde{x}^{i k}\right)-\left(A \tilde{x}^{i k}\right)^{\prime}\right)\left(e_{k}-e_{j}\right) \leq 0 .
\end{aligned}
$$

The final statement uses the fact that $V^{*}$ is $C^{1}$ on the boundary between the second and third cases of (52), the fact that $\left(v^{k i}\right)^{\prime} \tilde{x}^{i k}>0$, and the display before Lemma 4.1. 
For the third case of definition (52), we show that inequalities (20) and (21) hold at states $x^{*}, x^{i j}$, and $y^{j k} \in \ell^{i j}$, the last of which was introduced in the proof of Lemma 6.4 (Appendix A.3.2). The inequalities for the first two states are straightforward to check:

$$
\begin{aligned}
& D V^{3}\left(x^{*}\right)=D V^{3}\left(x^{*}\right)-\left(A x^{*}\right)^{\prime}=\mathbf{0}^{\prime} \text { (as a linear form on TX), } \\
& D V^{3}\left(x^{i j}\right)\left(e_{j}-e_{k}\right)=A^{j-k} x^{i j}>0 ; \\
& D V^{3}\left(x^{i j}\right)\left(e_{j}-e_{i}\right)=A^{j-i} x^{i j}=0 ; \\
& \left.D V^{3}\left(x^{i j}\right)-\left(A x^{i j}\right)^{\prime}=\mathbf{0}^{\prime} \text { (as a linear form on } T X\right) .
\end{aligned}
$$

It remains to check inequalities (20) and (21) for state $y^{j k}$. Since $D \chi^{i j}(x)\left(e_{j}-e_{i}\right)=0$,

$$
D V^{3}\left(y^{j k}\right)\left(e_{j}-e_{i}\right)=A_{j-i}^{j-i} d^{j i}\left(y^{j k}\right)>0 .
$$

Next, since

$$
d^{\zeta}\left(\chi^{j i}(x)\right)=d^{\zeta}\left(x+\left(e_{i}-e_{j}\right) d^{j i}(x)\right)=x_{k}^{*}-x_{k}
$$

and since for $y \in \mathcal{B}^{i j}$,

$$
\begin{aligned}
D \chi^{j i}(y)\left(e_{j}-e_{k}\right) & =\left(e_{j}-e_{k}\right)+\left(e_{i}-e_{j}\right) \frac{A_{j-k}^{j-i}}{A_{j-i}^{j-i}} \\
& =\frac{1}{A_{j-i}^{j-i}}\left(\left(e_{j}-e_{k}\right) A_{j-i}^{j-i}+\left(e_{i}-e_{j}\right) A_{j-k}^{j-i}\right) \\
& =\frac{1}{A_{j-i}^{j-i}}\left(A_{j-k}^{j-i} e_{i}+A_{i-k}^{i-j} e_{j}-A_{j-i}^{j-i} e_{k}\right) \\
& =\zeta^{i j},
\end{aligned}
$$

we have

$$
\begin{aligned}
D V^{3}(x)\left(e_{j}-e_{k}\right) & =A_{j-k}^{j-i} d d^{j i}(x)+A_{\zeta}^{\zeta} d^{\zeta}\left(\chi^{j i}(x)\right) D d^{\zeta}\left(\chi^{j i}(x)\right) D \chi^{j i}(x)\left(e_{j}-e_{k}\right) \\
& =A_{j-k}^{j-i} d d^{j i}(x)+A_{\zeta}^{\zeta}\left(x_{k}^{*}-x_{k}\right)\left(-e_{k}^{\prime} \zeta^{i j}\right) \\
& =A_{j-k}^{j-i} d d^{j i}(x)+A_{\zeta}^{\zeta}\left(x_{k}^{*}-x_{k}\right) .
\end{aligned}
$$

Thus the fact that $y_{k}^{j k}=0$ implies that 


$$
D V^{3}\left(y^{j k}\right)\left(e_{j}-e_{k}\right)=A_{j-k}^{j-i} d^{j i}(x)+A_{\zeta}^{\zeta} x_{k}^{*}>0
$$

This establishes the two cases of inequality (20) at state $y^{j k}$.

It remains to establish inequality (21) at state $y^{j k}$. Computing as above shows that for $y \in \mathcal{B}^{i j}$,

$$
\begin{aligned}
& D \chi^{j i}(y)\left(e_{i}-e_{k}\right)=\frac{1}{A_{j-i}^{j-i}}\left(\left(e_{i}-e_{k}\right) A_{j-i}^{j-i}+\left(e_{i}-e_{j}\right) A_{i-k}^{j-i}\right)=\zeta^{i j}, \text { and } \\
& D V^{3}(x)\left(e_{i}-e_{k}\right)=A_{i-k}^{j-i} d^{j i}(x)+A_{\zeta}^{\zeta}\left(x_{k}^{*}-x_{k}\right) .
\end{aligned}
$$

Hence

$$
\begin{aligned}
\left(D V^{3}(x)-(A x)^{\prime}\right)\left(e_{i}-e_{k}\right) & =A_{i-k}^{j-i} \frac{A^{j-i} x}{A_{j-i}^{j-i}}+A_{\zeta}^{\zeta}\left(x_{k}^{*}-x_{k}\right)-A^{i-k} x \\
& =\frac{1}{A_{j-i}^{j-i}}\left(A_{i-k}^{j-i} A^{j-i}-A_{j-i}^{j-i} A^{i-k}\right) x+\left(\zeta^{i j}\right)^{\prime} A \zeta^{i j}\left(x_{k}^{*}-x_{k}\right) \\
& =\frac{1}{A_{j-i}^{j-i}}\left(-A_{j-k}^{j-i} A_{i}-A_{i-k}^{i-j} A_{j}+A_{j-i}^{j-i} A_{k}\right) x+\frac{1}{A_{j-i}^{j-i}}\left(v^{i j}\right)^{\prime} \zeta^{i j}\left(x_{k}^{*}-x_{k}\right) \\
& =\left(v^{i j}\right)^{\prime}\left(\left(x_{k}^{*}-x_{k}\right) \zeta^{i j}-x\right) \\
& =\left(v^{i j}\right)^{\prime}\left(\frac{x_{k}^{*}-x_{k}}{x_{k}^{*}}\left(x^{i j}-x^{*}\right)-x\right) \\
& =\left(v^{i j}\right)^{\prime}\left(\frac{x_{k}^{*}-x_{k}}{x_{k}^{*}} x^{i j}-x\right) .
\end{aligned}
$$

The proof of Lemma 6.4 shows that $y^{j k}=x^{i j}+a\left(e_{j}-e_{i}\right)$ for some $a>0$. Thus since $y_{k}^{j k}=0$, we find that

$$
\left(D V^{3}\left(y^{j k}\right)-\left(A y^{j k}\right)^{\prime}\right)\left(e_{k}-e_{i}\right)=\left(v^{i j}\right)^{\prime}\left(y^{j k}-x^{i j}\right)=a\left(v^{i j}\right)^{\prime}\left(e_{j}-e_{i}\right)=a Q>0
$$

where the final equality follows from equation (36). This concludes the verification of the HJB equation at states where $V^{*}$ is smooth, and so completes the proof of Proposition 6.5. 


\section{References}

Alós-Ferrer, C. and Netzer, N. (2010). The logit response dynamics. Games and Economic Behavior, 68:413-427.

Benaïm, M., Sandholm, W. H., and Staudigl, M. (2014). Large deviations and stochastic stability in the large population limit. Unpublished manuscript, Université de Neuchâtel, University of Wisconsin, and Bielefeld University.

Blume, L. E. (1993). The statistical mechanics of strategic interaction. Games and Economic Behavior, 5:387-424.

Blume, L. E. (1997). Population games. In Arthur, W. B., Durlauf, S. N., and Lane, D. A., editors, The Economy as an Evolving Complex System II, pages 425-460. Addison-Wesley, Reading, MA.

Blume, L. E. (2003). How noise matters. Games and Economic Behavior, 44:251-271.

Boltyanskii, V. G. (1966). Sufficient conditions for optimality and the justification of the dynamic programming method. SIAM Journal on Control, 4:326-361.

Catoni, O. (1999). Simulated annealing algorithms and Markov chains with rare transitions. In Azéma, J., Émery, M., Ledoux, M., and Yor, M., editors, Séminaire de Probabilités XXXIII, pages 69-119. Springer, Berlin.

Dokumacı E. and Sandholm, W. H. (2011). Large deviations and multinomial probit choice. Journal of Economic Theory, 146:2151-2158.

Foster, D. P. and Young, H. P. (1990). Stochastic evolutionary game dynamics. Theoretical Population Biology, 38:219-232. Corrigendum, 51 (1997), 77-78.

Freidlin, M. I. and Wentzell, A. D. (1998). Random Perturbations of Dynamical Systems. Springer, New York, second edition.

Hofbauer, J. (1985). The selection mutation equation. Journal of Mathematical Biology, 23:41-53.

Hofbauer, J. and Sigmund, K. (1988). Theory of Evolution and Dynamical Systems. Cambridge University Press, Cambridge.

Kandori, M., Mailath, G. J., and Rob, R. (1993). Learning, mutation, and long run equilibria in games. Econometrica, 61:29-56.

Kandori, M. and Rob, R. (1995). Evolution of equilibria in the long run: A general theory and applications. Journal of Economic Theory, 65:383-414.

Kandori, M. and Rob, R. (1998). Bandwagon effects and long run technology choice. Games and Economic Behavior, 22:84-120. 
Myatt, D. P. and Wallace, C. C. (2003). A multinomial probit model of stochastic evolution. Journal of Economic Theory, 113:286-301.

Piccoli, B. and Sussmann, H. J. (2000). Regular synthesis and sufficiency conditions for optimality. SIAM Journal on Control and Optimization, 39:359-410.

Sandholm, W. H. (2001). Potential games with continuous player sets. Journal of Economic Theory, 97:81-108.

Sandholm, W. H. (2007). Simple formulas for stationary distributions and stochastically stable states. Games and Economic Behavior, 59:154-162.

Sandholm, W. H. (2009). Large population potential games. Journal of Economic Theory, 144:1710-1725.

Sandholm, W. H. (2010a). Decompositions and potentials for normal form games. Games and Economic Behavior, 70:446-456.

Sandholm, W. H. (2010b). Orders of limits for stationary distributions, stochastic dominance, and stochastic stability. Theoretical Economics, 5:1-26.

Sandholm, W. H. (2010c). Population Games and Evolutionary Dynamics. MIT Press, Cambridge.

Sandholm, W. H. and Staudigl, M. (2014). Stochastic stability in the small noise double limit, I: Theory. Unpublished manuscript, University of Wisconsin and Bielefeld University.

Schättler, H. and Ledzewicz, U. (2012). Geometric Optimal Control: Theory, Methods, and Examples. Springer, New York.

Staudigl, M. (2012). Stochastic stability in asymmetric binary choice coordination games. Games and Economic Behavior, 75:372-401.

Young, H. P. (1993). The evolution of conventions. Econometrica, 61:57-84.

Young, H. P. (1998). Individual Strategy and Social Structure. Princeton University Press, Princeton. 